\title{
TIME-DEPENDENT PROPAGATION OF HIGH-ENERGY LASER BEAMS THROUGH THE ATMOSPHERE: ॥
}

J. A. Fleck, Jr.

J. R. Morris

M. D. Feit

May 18, 1976

Prepared for U.S. Energy Research \& Deveiopment Administration under contract No. W-7405-Eng-48

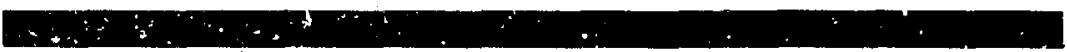

\section{UL LAWRENCE LVERMIOFE LABOAATOFY}

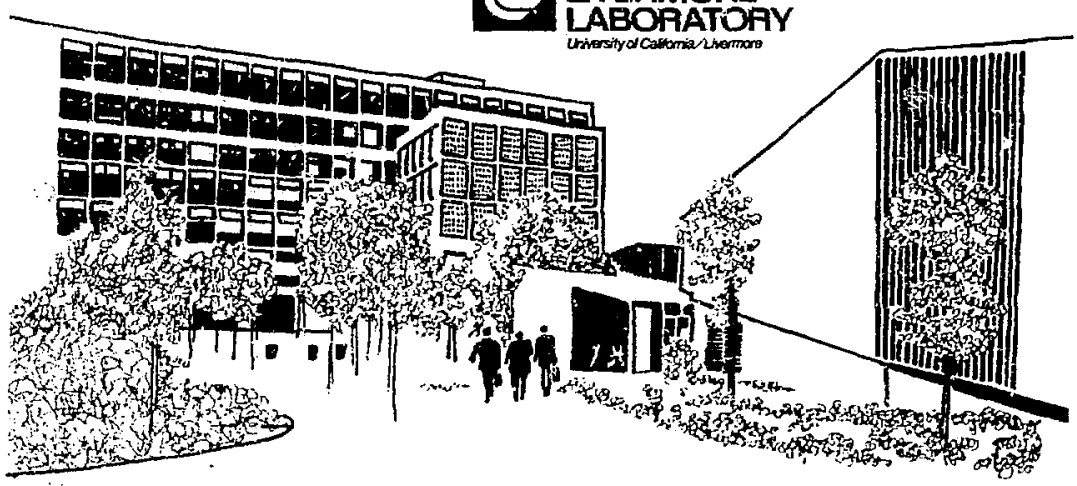




\section{NOTKE}

"Thb repiort whe prepated is in account of work oponcored by the Unired States Gownment. Neliber the United States nor the United States Entra Rexesch a bevelopmeat Administetion, not any of thel employtes, not any of theic contractors, subcontractori, or their employess, makes.rons vromenty, expes or implied, or axsumes ary lesal lability or repponsibility for the accuracy, completeness or unfutrets of eny information, uppesilus, proditet or proces: disclosed, of reptistents that its use would not in Trinse privhcely-owned rhats."

Printed in the United States of America Available from

National Technical Information Service U.S. Department of Commerce 5285 Port Boyal Road Springfeld, VA 22161 Price: Printed Copy S ; Microfiche \$2.25

\begin{tabular}{|c|c|c|c|c|c|}
\hline Pace Range & & $\begin{array}{c}\text { Domestic } \\
\text { Price }\end{array}$ & & Pege Renes & $\begin{array}{c}\text { Domintic } \\
\text { Prices }\end{array}$ \\
\hline $001 \cdot n 25$ & & s 3.50 & & $326-350$ & 10.00 \\
\hline $026-050$ & & 4.00 & 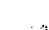 & $351-375$ & 10.50 \\
\hline $051-075$ &. & 4.50 & & $376-400$ & 0.75 \\
\hline $076-100$ & & 5.00 & & $401-425$ & 11,00 \\
\hline $101-125$ & & 5.25 & & $426-450$ & 11.75 \\
\hline $126-150$ & & 5.50 & & $451-475$ & 12.00 \\
\hline $151-175$ & & 6.00 & & $476-500$ & 12.50 \\
\hline $176-200$ & & 7.50 & & $501-525$ & 12.75 \\
\hline $201-225$ & & 7.75 & & $526-550$ & 13.00 \\
\hline $226-250$ & & 3.00 & & $551-575$ & 13.50 \\
\hline $251-275$ & & 9.00 & $\because$ & $576-500$ & 13.75 \\
\hline $276-300$ & & 9.25 & & 601-up & $\bullet$ \\
\hline $301-325$ & & 9.75 & & & \\
\hline
\end{tabular}

Add \$2.50 for each additional lio paze Inerement from 601 to 1,000 pages: add \$4.50 for jech additional 100 page increment over 1,000 pages. 


\title{
逭 \\ LAMPENCE LN:ERMORE LABORATORY \\ Universityou Callomo Livermore. Cattoma 94550
}

UCRI- -2071

\section{TIME-DEPENDENT PROPAGATION OF HIGH-ENERGY LASER BEAMS THROUGH THE ATMOSPHERE: ॥}

\author{
J. A. Fleck, Jr. \\ J. R. Lorris \\ M. D. Felt
}

MS. dace: May 18, 1976

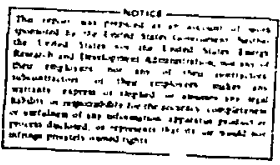




\section{Foreword}

Thta work was done under contract to the $t$. S. Navy, the Army Missile Comand, Hunteville, Alabama, and the U.S. Energy Research and Development Adoinistrat ion. 


\section{Contents}

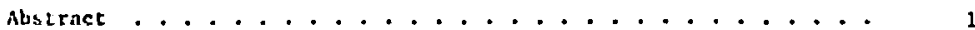

1. Introduction . . . . . . . . . . . . . . . . . . . . !

2. Treatment of Moving Stagnation Zones in Coplanar Scenarios . . . 9

3. Propagation of Mult spulse Laser Beams Through Seagnation Zones . . . . . . . . . . . . . . 10

4. E: fect of Longitudinal Afr Plotion on Flow in the Nelghborhood of a Stagnation Zone for Coplanar Scenarlo3 . . . . . . . . 16

5. Calculation of Transwerse Wind Velocitles for Noncoplanar Scenartos ........................... . . . 18

6. Sceady-State Solutions of Hydrodynanic Equations for Arbitrary Transverse Wind Velocities: cy Steady State . . . . 20

7. Steady-State Solutions of Hydrodynanic Equations for Arbitrary Transtverge Wind Velocities: Nultipulse Steady scate . . . . . . . . . . . . . . . . . . .

b. Bffect of Moncoplanarity on Propagation of $\mathrm{cw}$ Laser Beams Through Stagnation zones....................

7. Effect of Noncoplanarity of Propagation of Nultipulse Beams Througl Stagnation Zones....... . . . . . . . . . .

10. Single-Pulse Theranl Blooming in the Triangular Pulse Approxiantion . . . . . . . . . . . . . . . . . . .

11. Multipulae Therwal Bloonting in the Triangular Pulse

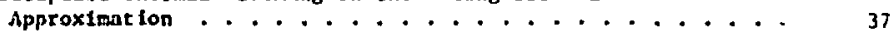

Acknowledgrent . . . . . . . . . . . . . . . . . . . . . . 43

References . . . . . . . . . . . . . . . . . . . . . . . 4 44

Appendix A: Adaptive Lens Transformation . . . . . . . . . . . . . . 45

Appendix $B:$ An Adaptive Algortchn far Selecting the Axial

Space Increnent .................... 53

Appendix C: Treatment of Multiline Absorption . . . . . . . . . . 56

Append IX D: Characterization of Nondiffraction-limited Bnans.... 59 


\section{TIME-DEPENDENT PROPAGATION OH HIGH-ENERGY LASER BEAMS THROUGH THE ATMOSPHERE: II}

\begin{abstract}
Various factors that can affect thermal blooming in stagnation zones are examined, including stagnation-zone motton, longitudinal atr motion in the neighborhood of the stagnation zone, and the effects of scenarto noncoglanarity. of these effects, only the last offers any reasonable hope of redusting the strong thermal blooming that normally accompanies stagnation zones; In particular, non-
\end{abstract}

Abstract coplanartey should benefit multipulse more than cw beams. The methods of treating nonhortzontal winds hydrodynamically for $c w$ and multipulse steady-state sources are discussed. Pulse "se1f-blooming" in the triangular pulse approximation is discussed in the context of both single and multipulse propagation. It is shown that se1F-blooming and wultipulse blooming cannot be treated Independently.

\section{Introduction}

This is the second report in a sertes dealing with the general problem of time-dependent thermal blooming of multipulse and $c w$ laser beams. ${ }^{1}$ Ttme dependence is essenttal for describing the propagation of laser beams through stagnation zones, which are created whenever the motion of the laser platform and the slewing of the laser beam combine to create a null effective transverse wind velocity at some location along the propagation path. The location of vanishing transferse wind we sha11 call the stagnation point, and the term stagnation zone will refer to the portion of the propagation path, extending in both dire:tions from the stagnation point, where the transverse wind has not yet had time to blow completely across the beam. The lack of wind at the stagnation point creates a stead $11 y$ decreasing density and a thermal lens whose strength grows with time. This report will continue the study of stagnation 
zones begun in Ref. 1, and discuss contributions of self-blooming to multipulse thermal blooming and new models that lave been added to the Four-D code.

Both pivoted-absorption-cell measurements ${ }^{2,3}$ and detalled numerical calculations of the experimental arrangements ${ }^{1,4}$ give evidence that the blooning effects of stagnation zones tend to saturate with time. Thus the tieam characteristics seem -o approach a kind of quas1-steady stace, which is posstbly a result of the steady reduction in length of the stagnation zone with time. ${ }^{3}$ Despice the existence of these quast-steady stutes, calculactons for high-power beams show that stagnation zones can lead to gevera beam iegradution.

The notion of a stagnation zone requires that che transverse wind velucity vanish at at least one position along the propagation path. There are always present, however, a number of addicionil effects that w111 prevent a completely stagnant wind cosdition from occurring at any position. These effects are:

1. latural convection

2. Motion of the stagnation point with time

3. Longttuditnal afr mottan at the stagnation point

4. Vertical alr motion due to noncoplanar scenario geometry
A realistic appraisal of the influence of stagnation zones on bizam propagation requires that each of these effects be assessed and posstbly Incorporated Into the computatlonal model.

Natural convestion flow at the stagnation point should be negligjbla for practical beam sizes. A 3.8-jm wavelength and a $474-\mathrm{kW}$ beam power, for example, give a natura! convection velocity of the order of $10 \mathrm{~cm} / \mathrm{s} .1$ For this flow veloctiy and a beam radius of $10 \mathrm{~cm}$ at the stagnation point, ap proximately 2 s is required for the beam to approach a steady-state density distribution. This time is axcessive for preventing or reducing stagnation-zone blooming tffects, whll may develop in times r. : ing from 1 ins to $0.1 \mathrm{~s}$. At a 10.6-jm wavelength the laser heating rate of the atmosphere is somewhat greater for the sane intensity, but the natural convestion veloctty scales only with the cube root of the absorbed power, Bo flow veloctties would not be sigulficantly above those for the 3,8-jm case, Mere.. fore, we shall not consider the effects of natural. convection further.

Under most conditions the stagnation point is not stationary but moves tn the sami general direction as the target with a velocity that is little different from the 
target's. The parcel of air that sees a null wind speed changes with time and thus does not heat up in the manner of a stationary parcel. The influence of this stagnationpuint motion on beam propagation was found to be minimal for a cw waveform example truated in Ref. 1. Stagnation-point motion dlso turns out to be unimportant for a multipulse scenario examined in this report. The concluston is that stagnation-point motion is unlikely to have much, if any, effect in alleviating stagnation-zone blooming, since, despite the motion, a substantial propagation path exists over which wind velocities are negligible.

The existence of a null transverse wind-velocfty component at a stagnation polnt in no way guarantees a vanishing magnitude of the wind vector, because a nonvantshing longtcudinal component almost always exists there. Any air parcel found within the beam at the stagnation point will, as a result, exit from the beam in a finite length of time. Indeed, In coplanar geometries all wind-flow trajectories should cross the beam in two locations: one for values of $z$ (longitudinal position) below the stagnation point $z_{B}$, and the other for values above $z_{\beta^{*}}$. The wind flow may be in either the positive- or negative-z direction. In the neighborhood of the stegnation point, the wind-flow trajectories -111 enter one side of the beam, reverse direction with the beam, and exit on the same side. 5 The restdence time in the beam for flutd parcels passing through the beam center at the stagnation polst $\mathrm{Hll}$, of course, depend on scenario paramteters, but for some typical beam sizes and scenarios this time can be of the order of 0.5 to $1 \mathrm{~s}$. Longttudinal flow should thus be as effective as natural convection in controlling density changes at the staznation point. One consequence of these re-entrant wind-flow trajectortes is that air densteties for $z$ values greater than $z_{a}$ could be influencel hydrodynamically by densitles for $z$ values less than $z_{b}$; but for any foreseeablt practical scenarios, the re-entrant times except perhaps in the Inmediate netghborhood of the stagnation polnt - would be considerably loz.ger than times of interost. ConsequentIy, hydrodynamic coupling between points above ind below $z_{s}$ can be safely neglected in cases of praitical interest.

The existence of a position where the transverse wind velocity vanishes presupposes the extremely Improbable. coplanartity of the laser beam and the trajectorles of 1 ts platform and the target - a situation that is clearly a limiting case of real-world 
Ecenarios, which are invariably noncoplazar. In the more general case of noncoplanar geometry, only the wind component along a certain transverse axls can be expected to vanish. The wind vector in the transverse plane w11 rotate and attain 1 ts minimum magnitude at the stagnation point. Since this minimum magnitude can zever vanish, except in a space of measure zero, a steady state can always be defined for the governing hydrodynamic equations. The signifIcance of this is that, In systems analysis, steasy-state numbers can always be assigned to stagnation-zone situations, at least f.r some nominal degree of nonsoplanerity, and these numbers can be obtained from simple and relatively cheap steady-state calculations. Truly coplanar stagnation-zone situations, in contrast, require time-dependent calculations that are expensive and require comsiderable care in execuition.

In the coplanar scenario described In Ref. 1, for example, if the laser is given an elevation of $10 \mathrm{~m}$ abcve the plane containfing the $t$ arget and the laser platform, the vertical component of wind velocity at the stagnation point takes on the value of $1 \mathrm{ai} / \mathrm{s}$. This $1 \mathrm{~s}$ sufficient to establish a steady state in a time of tice order of $0.1 \mathrm{~s}$, which is short compared to times of interest. The small vertical velocity compone it at the stagnation point leads to substantial changes in isointensity contours in the fecal plane, but the average Intensity is remarkabiy close to the quasi-steady-state value obralned in a time-dependent calculation for the corresponding coplanar case.

Thus, small amounts of noncoplanarity should not be expected to greatly improve cw laser performance in stagnation-zone situations but should contribute ro ease in understanding and predicting 1 t. The case of multipulse beams is another matter. As pulse-repetirton frequeristes are lowered, a small vertical wind component at the stagnation polnt becomes more and more effoctive in sweeping out the air between pulses. The banefits of noncoplanaricy in stagnazion-zone sttuations should thus be greater for multipuis: beams than for $\mathrm{cw}$ beans.

The current status of the Four-b code is summarized in Table 1 , and recent additions to the code are described in the body of this report. We have continued to adhere to the philosophy that the best way to approach all laser-propagation calculations is chrough is single, unified computer code that can be applied to any probler. The advantages of this are chreefold. First, it greatly simplifies bookkeeping (or, 
more appropriate1y, code-keepiug), since a proliferation of 1 imited special-purpose codes is avolded. Second, if each type if calculation Is made is subset of a larger calculational capability, new features added to the code - such as data- processing routines, adaptive-lens transformations, scenario features, etc. - are avallable to all typeg of calculation at once. Third, realistic simulations are posstble, since a wide range of conditions can be incorporated into any calculation.

Table 1. Bastc out 1ine of current Four-D propagation code.

Variables

Form of propagation equation

Method of solving propagation equation

Hydrodynanies for steady-state cw problems

Transonic slewing $x, 2,2, t$, where $x$, ar transverse coordinares and $a$ is axtal displacement.

Scalar wave equation in parabolic approxilmation

$$
2 \because k \frac{\partial \varepsilon}{\partial z}=\nabla_{1}^{2} \varepsilon+\dot{\gamma}^{2}\left(r^{2}-1\right) \varepsilon .
$$

Svgmetrized split operator, finite Fovrier series, fast Fourier transform (FFT) algorithm

$$
\begin{aligned}
& \varepsilon^{n+1}=\exp \left(-\frac{i \Delta z}{42} \nabla^{i} j \exp \left(-\frac{i \Delta z}{2 k} \bar{x}\right)\right. \\
& \exp \left(-\frac{i \Delta a}{4 k} \nabla_{1}^{2}\right) \varepsilon^{\gamma} \\
& x=i^{2}\left(n^{2}-1\right) .
\end{aligned}
$$

Uses exact solution to l1near hydrodynamic equations. Fourfer method for $M<1$. Characteristic mechod for $M>1$. Solves

$$
\begin{aligned}
& v_{z} \frac{\partial \rho_{1}}{\partial x}+v_{y} \frac{\partial \rho_{1}}{\partial y}+\rho_{0} \underline{\nabla}_{1} \cdot \underline{v}_{-}=u, \\
& \rho_{v}\left(v_{x} \frac{\partial v_{1}}{\partial x}+v_{y} \frac{\partial v_{1}}{\partial y}\right)+\underline{\nabla}_{1} p_{1}=0, \\
& \left(v_{x} \frac{\partial}{\partial x}+v_{y} \frac{\partial}{v_{y}}\right)\left(p_{1}-c_{s}^{2} \rho_{1}\right)=(\gamma-1) \simeq \tau .
\end{aligned}
$$

Steady-state calculation valid for all Mach numbers except $M=1$. Code can be used arbitrarily close to $M=1$. 
Treatment of stagnation-zone problems for $\mathrm{cw}$ beams

Nonsteady treatment of mult1pulse density changes

Method of culculcting density change for individual pulse in train

Treatment of steady-state multipulse blooming
TIme-dependent isobaric approximation. Transient succession of steady-state density changes; 1.e., solves

$$
\frac{\partial \rho_{1}}{\partial t}+v \frac{\partial \rho_{1}}{\partial x}=-\frac{\gamma-1}{c_{s}^{2}} \alpha I \text {. }
$$

Changes in density from previous pulses in traln are calculated wi.th isobaric approximation using

$$
\begin{aligned}
& \frac{\partial \rho_{1}}{\partial t}+v_{x} \frac{\partial \rho_{1}}{\partial x}= \\
& \quad-\frac{y-1}{c_{s}^{2}} \sum_{n} \tau I_{n}(x, y) \delta\left(t-t_{n}\right) .
\end{aligned}
$$

where $\tau I_{n}(x, y)$ is $n$th pulse fluence. Dansity changes resulting from the same pulse are calculated using acoustic equations and trlangular pulae shape.

Takes two-dimens:onal Fourler transform of

$$
\frac{\alpha \tilde{r} t_{p}}{2 c_{s}^{2}}\left\{1-\frac{\sin ^{2}\left[\frac{I_{c}}{2} c_{s}\left(k_{2}^{2}+k_{y}^{2}\right)^{1 / 2} t_{p}\right]}{\left[\frac{1}{2} c_{s}\left(k_{x}^{2}+k_{y}^{2}\right)^{1 / 2} t_{p}\right]^{2}}\right\}
$$

where $\tilde{I}$ is Fourier transform of Intensity, and $t_{p}$ is the time duration of each pulse. Source aperture should be softened shen using this code provision.

Previous pulses in traln are assumed to be periodic replications of current pulse. Solves

$$
\begin{aligned}
\frac{\partial \rho_{1}}{\partial t}+v_{x} \frac{\partial \rho_{1}}{\partial x} & +v_{y} \frac{\partial \rho_{1}}{\partial y} \\
& =-\frac{Y-1}{c_{s}^{2}} I \sum_{n} \delta\left(t-t_{n}\right) .
\end{aligned}
$$

Pulse self-blooming is treated as in the nonsteady-state case.

Uses phase-screen method of Bradley and Brown wth Von Karman spectrum. Phase screen determined by 


$$
\begin{array}{r}
\Gamma(x, y)=\int_{-\infty}^{\infty} \mathrm{d} k_{x} \exp \left(i k_{x}\right) \int_{-\infty}^{\infty} \mathrm{d} k_{y} \exp \left(i k_{y}\right) \\
\times a\left(k_{x}, k_{y}\right) \Phi_{n}^{1 / 2}\left(k_{x}, k_{y}\right),
\end{array}
$$

where $a$ is a complex random variable and $\Phi_{n}$ ts spectral density of index fluctuations.

Lens transformation and treatment of lens optics

Treatment of nondiffraction limited beams

Adaptive lens transformation
Compensates for a portion of lens phase front with cyllndrical Talanov lens transformation. Uses in sphericai case

$$
\frac{1}{z_{f}}=\frac{1}{z_{T}}+\frac{1}{z_{L}},
$$

where $a_{f}$ is focal length of lens, $z_{T}$ is focal length compensated for by Talanov transformation, and $a_{L}$ is focal length of Initial phase front.

Spherical-aberration phase determined by

$$
\phi^{S A}=\frac{2 \pi A}{\sigma^{2}}\left(x^{2}+y^{2}\right)^{2} \text {. }
$$

or pliase-screen method of Hogge et ci. Phase determined as in turbulence, only

$$
\phi_{n}=\frac{\sigma^{2} z_{0}^{2}}{2 \pi} \exp \left(-\frac{i_{0}^{2} k^{2}}{2}\right)
$$

where $z_{0}$ is correlation length and $c^{2}$ phase variance.

Removes phase

$$
\sum_{i=1}^{2}\left[\alpha_{i}\left(x_{i}-\left\langle x_{i}\right\rangle\right)^{2}+\beta_{i}\left(x_{i}-\left\langle\omega_{i}\right\rangle\right)\right]
$$

through lens transformation and deflection of beam. Here $x_{1}=x, x_{2}=y$, averages are Intensicy weighted, $\alpha_{i}$ and $\beta_{i}$ are calculated to keep the intensity centrofi at mesh center, and intensity weighted r.m.S. values of $x$ and $y$ are constant with $z$. 
Table I (continued).

Selection of $z-s$ tep

Adaptive z-step selectlon bas 1 on limiting gradients In nonifnear contribution to phase. Constant z-step over any portion of range also possible.

Scenarto capability

Treatment of multiline effects

Treatment of beam jitter

Code output

Numerical capacity when used with CDC 7600 and restricted to internal memory (large and small core)

Problem zoning features
General noncoplanar scenarlo geometry capablity involving troving laser platform, moring target, and arbitrary wind direction. In coplanar case, wind can be function of $t$ and $z$.

Calculates average absorption coefficient based on asgumption of Identical fleld distributions for all lines

$$
\bar{\alpha}=\frac{\sum_{i} \alpha_{i} f_{i} \exp \left(-\alpha_{i} z\right)}{\sum_{i} f_{i} \exp \left(-\alpha_{i} z\right)}
$$

where $f_{i}$ is fraction of energy in line $i$ at $z=0$.

Takes convolutim of intensity in target plane with Gaussian distribution:

$$
\begin{aligned}
I_{\text {jltter }}=\iint & \exp \left(-\frac{x^{\prime 2}+y^{\prime 2}}{2 \sigma^{2}}\right) \\
& \times I\left(x-x^{\prime}, y-y^{\prime}\right) d x^{\prime} d y^{\prime},
\end{aligned}
$$

where $\sigma^{2}$ is variance introduced by jitter.

Isolntensity, 1sodensfty, isophase, and spectrum contours. Intensity averaged over contours. Plots of intensity, phase, density, spatial spectrum along specific directions, etc, at spectflc times.

Plots of peak intensity and average intensity vs time.

Spatial mesh, $64 \times 64,35$ sampling times, no restriction on number of axial space increments.

Number of space increments in $x$ and $y$ directions must be equal and expressible as a power of 2 . 


\section{Treatment of Moving Stagnation Zones in Coplanar Scenarios}

The basic coplanar scenarto geometry is depicted in Fig. 1. It is assumed that the target and laser platform will collide on the $x$ axis after a time $\tau_{c}$ has elapsed. The point of impact is denoted by $P$ and the position of the laser by $L$. The effective transverse wind speed $v_{t}$ Is given by the expression

$$
\begin{aligned}
& V_{t}\left(\tau_{e}, z\right)=-\sin \theta_{T}\left[V_{T}\right. \\
& \left.-\frac{z}{R}\left(V_{T}-V_{P}+V_{n} \cot \theta_{T}\right)\right] \\
& \quad+V_{W} \sin \left(\theta_{W}-\theta_{T}\right),
\end{aligned}
$$

where $V_{R}$ is the target (recelver) velocity, $V_{T}$ is the laser-platform (transmitter) velocity, $V_{V^{\prime}}$ is the background wind velocity, $R$ is the

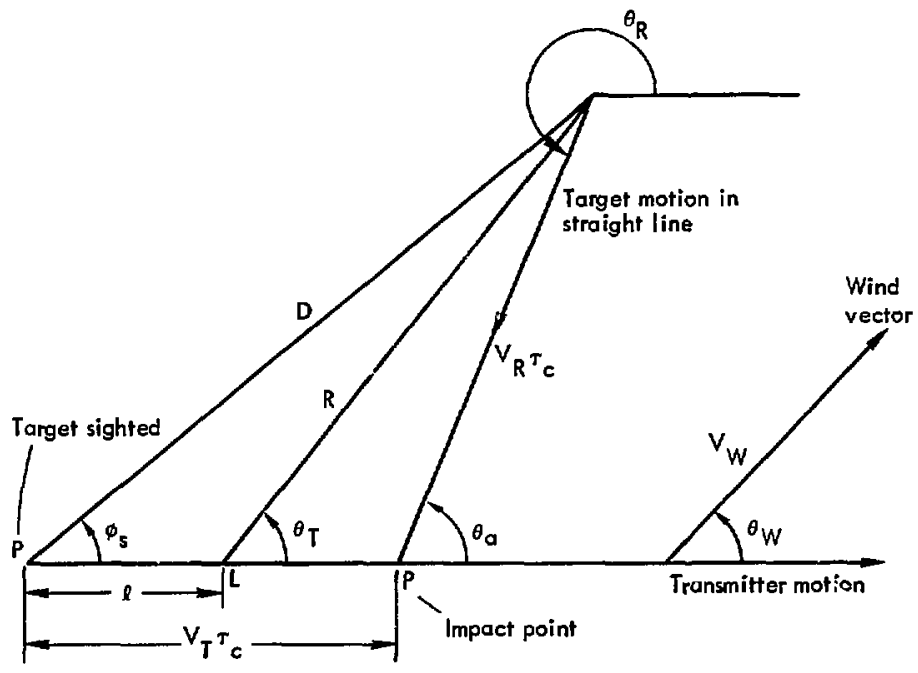

Fig. 1. Diagram of coplanar scenario model. 
range,

$$
V_{p}=V_{R} \cos \theta_{R},
$$

and

$$
V_{n}=V_{R} \sin \theta_{R}
$$

From simple trigononetry,

$\theta_{T}=\cot ^{-1}\left[\frac{\cos \theta_{a}+\left(V_{T} \tau_{c}-\tau\right) / V_{R} \tau_{c}}{\sin \theta_{a}}\right]$

$R=\frac{D \sin \phi_{B}}{\sin \theta_{T}}$,

where $\theta_{a}$ is constant.

The transverse wind speed $V_{t}$ depends on $\tau_{c}$ and hence on time through the dependence of the scenario parameters $\theta_{T}$ and $R$ on time In Eqs. (4a) and (4b). The Four-D code is programmed to calculate $V_{t}\left(\tau_{c}, z\right)$ as a function of $\tau_{e}$ and hence of time. The hydrodynamic equations are solved numerically by assuming that $V_{t}$ is stepwise constant over each Integration time interval $\Delta t$.

The location of a stagnation point is determined by setting the righthand side of Eq. (1) equal to zero. clearly, that point moves with time, and, as a result, a different parcel of alr undergoes heating under conditions of zero wind velocity at each instant. In general the stagnation point will move with a velocity comparable to that of the target. The determination of $V_{t}$ from Eqs. (1)-(4) for use in the hydrodynamic equations permits an accurate determination of the effect of motion on the thermal lens in the stagnation zone. It is more difficult to follow the Irradiance on a moving target, however, since all equations are solved in a retirded time frame. It would be necessary to atore the Irradiance history for the values of $z$ corresponding to the tareet motion. Since the relative change of the range $R$ in a time of interest is small, it is a good approximation to assume the focal distance and the range $R$ at which the laser intensity Is monitored to be constants in time, while tis correct varlable $R$ is taken Into account in the hydrodynamic portion of the calculation by means of Eqs. (1)-(4).

\section{Propagation of Multipulse Laser Beams Through Stagnation Zones}

The partlcular scenarto chosen leads to the effective transverse wind as a function of range for $\tau=0$, shown in FIg. 2, where $\tau$ is measured from the time the pulse turns on. The pertinent physical 
data are the following:

Power, $P$

$53 \mathrm{~kW}$

Range, $R$

$2.5 \mathrm{~km}$

Focal length, $f$ $4.5 \mathrm{~km}$

Absorption coefficlent, $\alpha 0.25 \mathrm{~km}^{-1}$

Wavelength, $\lambda$

$10.6 \mathrm{\mu m}$

Aperture diameter, $2 a$

(Gaussian at $1 / e^{2}$ )

$30 \mathrm{~cm}$

slewing rate, $\Omega$

7.44

$\mathrm{mrad} / \mathrm{s}$

Pulse-repetition rate, $v 10,25,50$,

These data can be expressed in terms of the following dimensionless numbers:

$N_{F}=k a^{2} / f=2.96$,

$N_{0}=2 a / v_{0} \Delta t=3.2 \times 10^{-2} v$,

$N_{S}=\Omega f / v_{0}=3.6$,

$N_{A}=\alpha f=1.125$,

$N_{D}=\frac{1}{2} \frac{\partial \varepsilon}{\partial \rho} \frac{N_{0} \nu_{A} N_{F}(\gamma-1) E_{p}}{c_{s}^{2} a^{3}\left(\frac{a}{f}\right)}=100$,

where $N_{F}, N_{O}, N_{S^{\prime}} N_{A}$, and $N_{D}$ represent respectively the Fresnel, overlap, slewing, absorption, and distortion numbers. Although the chosen power, $53 \mathrm{~kW}$, is rather low, the value of the discortion number $N_{D}$ is quite high, and the resulting theras bloowing is about the maximum that the code can accommodate. In any case. the above parameters

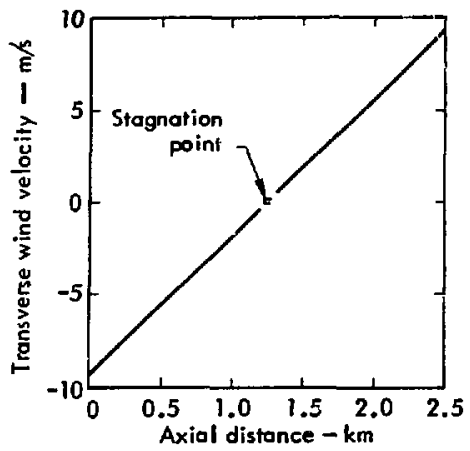

Fig. 2, Transverse wind velocity as a function of axtal distance.

are adequate for assessing the sensitivity of typical multipulse laser performance to stagnation-zone motion.

In the present scenario, the stagnation zone and target move with a speed of $300 \mathrm{~m} / \mathrm{s}$. For a mulctpulse beam with $v=100 \mathrm{~s}^{-1}$, the stagnation zone moves 3 m between pulsea, whereas for $v=10 \mathrm{~s}^{-1}$, the stagnation zone moves $30 \mathrm{mbetween}$ pulses. It would be hoped that, in the case of the lower pulserepetition frequency, the greater movement of the stagnacion zone wouid lead to a reduced butldup of stagnant-afr density changes. This effect turns out to be minimal. The time dependence of the average intensity on target (averaged over the minimum half-power area) is shown for the case of no stagnetionzone motion In FIg. 3(a) and for the 


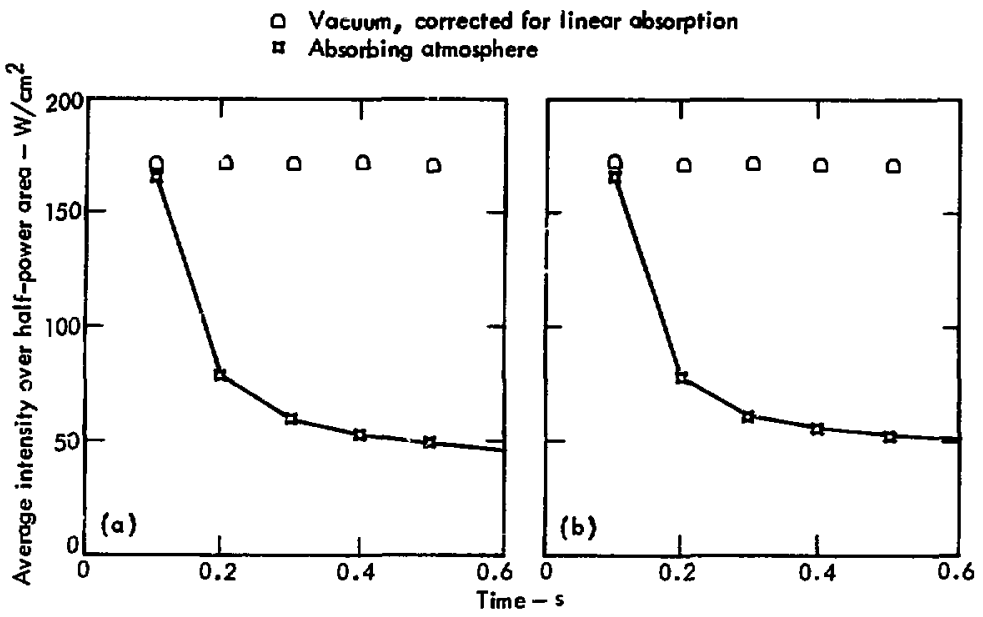

Fig. 3. Area-averaged target intensity as a function of pulse time: six pulses at $V=10 \mathrm{~s}^{-1}$. (a) No stagnation-zone motion included. (b) Stagnation-zone motion included.

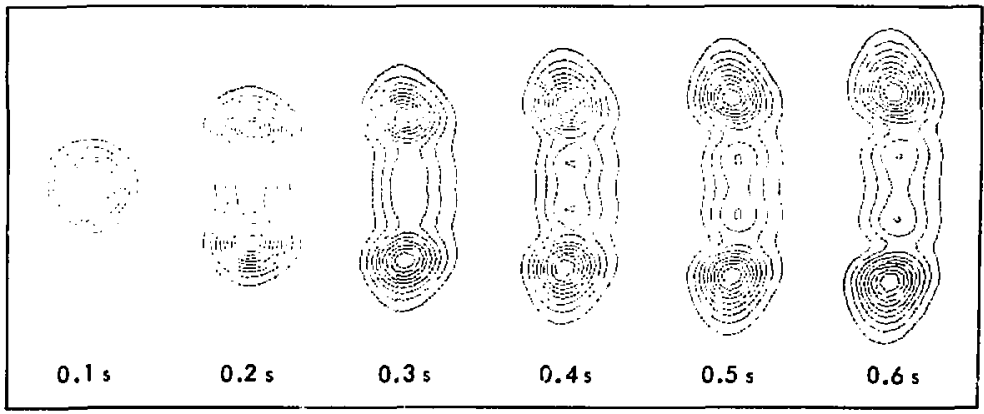

Fig. 4. Is: Litensity contours as a function of pulse time for $v=10 \mathrm{~s}^{-1}$. 
Table 2. Comparison of multipulge Intensities with and without stagnationzone motion.

\begin{tabular}{|c|c|c|c|c|}
\hline \multirow{3}{*}{$\begin{array}{l}\text { Time } \\
\text { (s) }\end{array}$} & \multicolumn{4}{|c|}{ Time-averaged Intensity $\left(\mathrm{W} / \mathrm{cm}^{2}\right)$} \\
\hline & \multicolumn{2}{|c|}{ No mol Lon } & \multicolumn{2}{|c|}{ Motion } \\
\hline & Average & Peak & Average & Peak \\
\hline 0.1 & 168.5 & 234.0 & 168.5 & 234.0 \\
\hline 0.2 & 78.9 & 117.3 & 78.7 & 117.3 \\
\hline 0.3 & 60.7 & 100.7 & 60.8 & 103.3 \\
\hline 0.4 & 53.7 & 92.5 & 55.3 & 96.8 \\
\hline 0.5 & 49.6 & 88.7 & 52.4 & 92.7 \\
\hline 0.6 & 47.3 & B4.1 & 50.2 & 87.5 \\
\hline
\end{tabular}

Maximun increase resulting from stagnation-zone motion: av, 6.1\%; peak, $4.0 \%$.

case with stagnation-zone motion in Fig. 3(b). The calculation is carrled out for six pulses. The isointensity contours for the slx pulses are shown in Fig. 4 for the moving stagnation zone. The contours in the nonmoving case are so similar that they are not shown. The performance in the two cases is summarized pulse by pulse in Tahle 2, where the intensity values have been averaged over the Interpulse separation $t$ ime, and the percent Improvements in incensity indicated are for the last pulse in the tratn.

Surprisingly, the Improvements in peak and average fluence go in the opposite direction. The peak and average fluences are actually slightly higher in the no-motion case, as sñown 1 a Table 3 .

This behavior is due to the large contribution that the first pulse in the train makes to the total fluence. The Isofluence contours for the case with motion are displayed in Fig. 5. The central

fluence peak contains the maximun value and makes the largest contribution to the fluence averaged over the minimum half-power area. Apparently in the no-motion case the subsequent pulses 1 !: the train make a greater contribution in the central region than do the corresponding pulses in the case with motion. This small difference in peak and average fluences is of

Table 3. Comparison of fluences with and without stagnation-zone motion.

\begin{tabular}{cccc}
\hline \multicolumn{4}{c}{ Fluence $\left(\mathrm{J} / \mathrm{cm}^{2}\right)$} \\
\hline \multicolumn{2}{c}{ No motion } & \multicolumn{2}{c}{ Motion } \\
\hline Average & Peak & Average & Peak \\
\hline 30.61 & 42.1 & 30.1 & 40.7
\end{tabular}

Decrease resulting from stagnationzone motion: av, $1.7 \%$; peak, $3.3 \%$. 


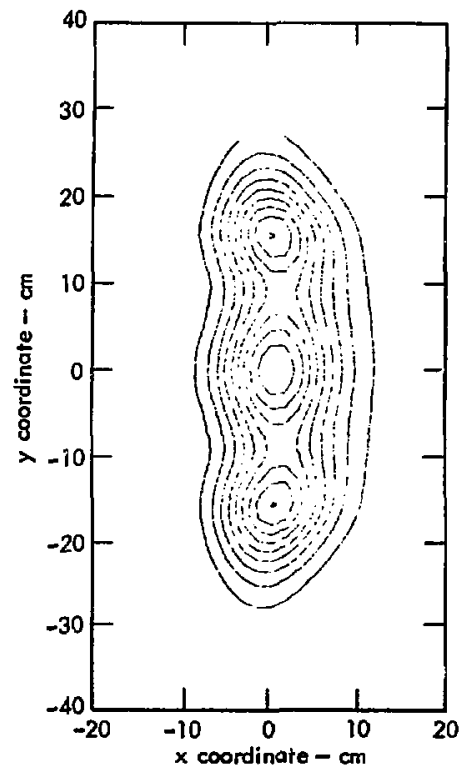

Fig. 5. Fluence contours for case of $v=10 \mathrm{~s}^{-1}$, motion included.

little or no practical importance, and is Indicative of the fact that stagnation-zone motion plays no vital role in determining thermal blooming in stagnation zones.

FIgure 6 shows the dependence of average intensity on time at the terget range for different values of pulse-repetition rate, $v$. Each curve beging with the time of arrival of the second pulse. (The first pulse wuld create a timeaveraged Intensity of $189 \mathrm{w} / \mathrm{cm}^{2}$.) It is clear that reducing $v$ diminishes the effact of the stagnation zone. The reason obviously is that for smaller values of $v$ the air can be swept out by wind between pulses over a greater proportion of the propagation path.

Sample pulse-1solntensity contours for $v=100 \mathrm{~s}^{-1}$ are displayed in F1g. 7; these should be compared with those for $v=10 \mathrm{~s}^{-1}$ in Fig. 4 . At the lower repetition rate, the beam has divided into two distinct spots. At the higher rate, lateral peaks are also formed but they are much less distinct. The lateral spreading of the contours as a function of time is shown in Fig. 8 . The width perpendicular to the wind

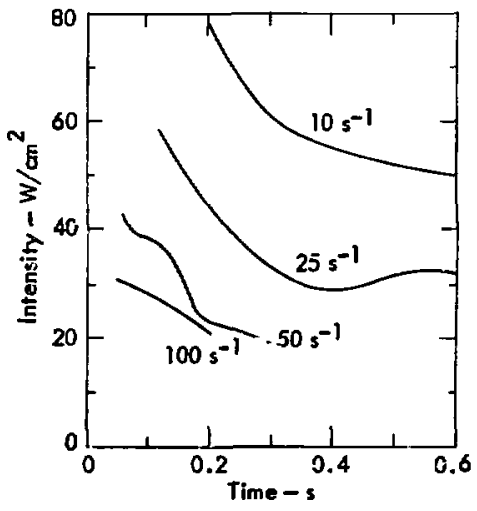

Fig. 6. Space-averaged intensity as a function of pulse time for vartous pulse-repetition rates. 


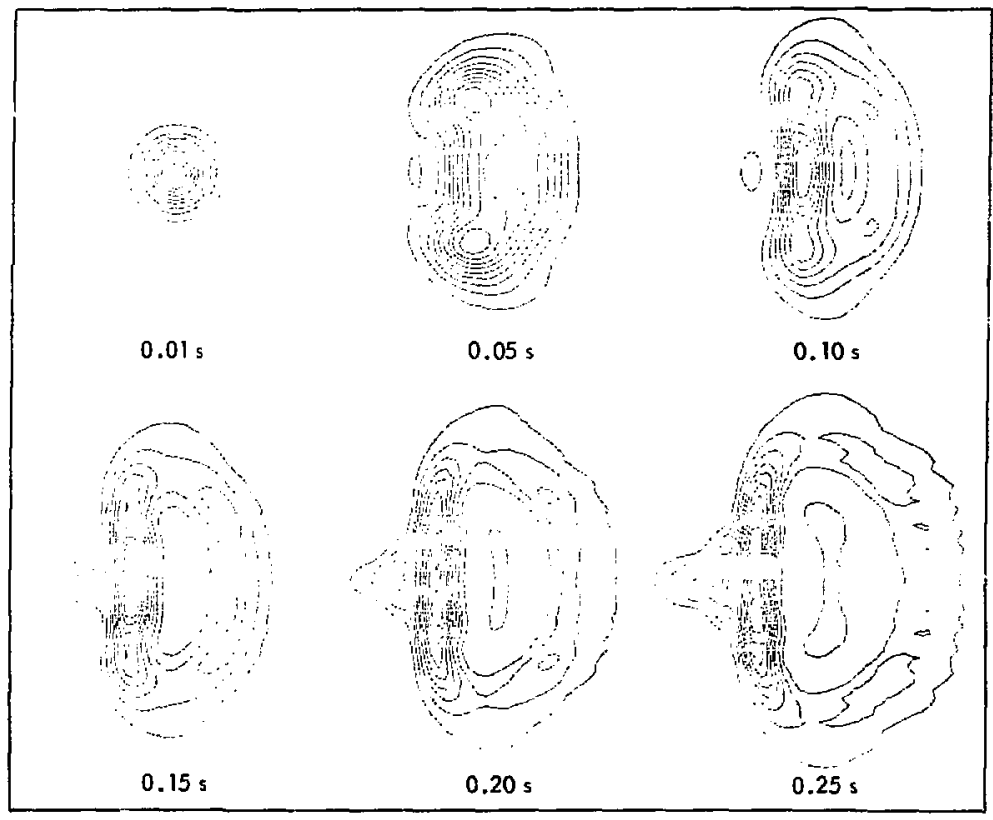

Fig. 7. Isolntensticy contours for pulses sampled from $v=100 \mathrm{~s}^{-1}$ train.

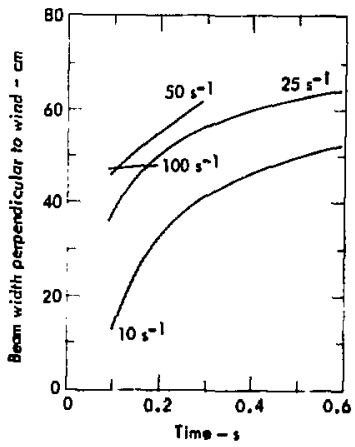

Fig. 8, Width of beam in direction perpendicular to the wind at varlous pulse-repetition frequencies. 
is determined by measuring the maximum distance perpendicular to the wind direction bistween $30 \%$ contours.

In conclusion, the performance of a multipulse laser under stagnation-zone conditions can be Improved by lowering the pulserepetition Erequency, but, witil or without motion of the stagnation point, the thermal blooming is likely to be substantial.

\section{Effect of Longitudinal Air Motion on Flow in the Neighborhood of a Stagnation Zone for Coplanar Scenarios}

We wish to examine the air-flow trajectories in a coordinate system that moves with the laser beam. Take the $x$ axis along the direction of motion of the laser platform and the $y$ axis perpendicular to it in the scenarto plane. The unit vector $\hat{z}(t)$ is directed along the rotating laser beam, and $\hat{x}^{\prime}(t)$ is taken normal to $\hat{z}(t)$ (see Fig. 9). Ai any instant of time, $\hat{z}(t)$ and $\hat{x}^{\prime}(t)$ can be expressed in the rest frame of the laser platform by means of the relations

$\hat{z}(t)=\left[\cos \theta_{T}(t), \sin \theta_{T}(t)\right]$,

$\hat{x}^{\prime}(t)=\left[\sin \theta_{T}(t),-\cos \theta_{T}(t)\right],(5 b)$

where

$\theta_{T}=\cos ^{-1}(\hat{A} \cdot \hat{x})$,

and the angle $\theta_{T}$ is calculated from the scenarto Eq. (4a) by subst 1tuting $\tau_{0}-t$ for $\tau_{0}$. The common origin for the rest frames of the laser platform and the rotating laser beam will be taken at the center of the laser aperture.

The effective wind vect, $r$ in the moving coordinate system of the laser can be expressed as

$$
\begin{aligned}
& \underline{\mathbf{v}}_{\text {eff }}=\underline{\mathbf{v}}_{\mathrm{rel}}-\frac{\left(\underline{\underline{v}}_{R}-\underline{\mathrm{v}}_{T}\right)}{R(t)} \cdot z^{\prime} \\
& \times\left[z(t) \hat{x}^{\prime}(t)-x^{\prime}(t) \hat{z}(t)\right] .
\end{aligned}
$$

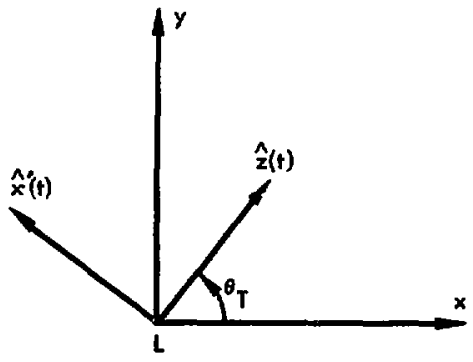

Fig. 9. Vector diagram in scenario plane; $\hat{\boldsymbol{z}}(t)$ Indicates Instantaneous direction of slewting laser beam. 
Here $\underline{v}_{R}$ is the target veloctty relative to the earth's surface, and

$$
\underline{v}_{\text {rel }}=\underline{v}_{w}-\underline{v}_{T},
$$

where $V_{T}$ is the veloctty of the laser platform and $\underline{v}$, is the veloc1ty of the wind. An air test particle will move in the rest frame of the laser platform along trajectortes described by

$$
\underline{r}(t)=\underline{r}(0)+\underline{v}_{r e I} t .
$$

These trajectories can be expressed In the rotating frame of the laser beam by means of the following relations:

$$
\begin{aligned}
& x^{\prime}(t)=\underline{r}(t) \cdot \hat{x}^{\prime}(t), \\
& z(t)=\underline{r}(t) \cdot \hat{z}(t) .
\end{aligned}
$$

Figure 1.0 shows sample airparticle trajectorles in the vicinity of a stagnation point for four different scenarlos of pracelcal interest cof the type show in
Fig. 1). The target range and stagnation point location at $t=0$ are Indicated in Table 4. At time $t=0$ the particles are assumed to be located pracisely at the stagnation point. The origin of the transverse coordinate is assumed to be at the center of the heam. The ticks on the trajectories indicate points separated by $0.5 \mathrm{~s}$ in tine. The arrows indicate the direction of air flow with increasing time. Also shown in Table 4 are the times $\tau$ actually spent in the laser beam by a particle that crosses the stagnation point at the center of a 10-cri-radius beam.

The longltudinal wind speed in the nelghborhood of the stagnation point is roughly equal to $\underline{v}_{r e l}$, as can be seen from Eq. (7). For the scenarios described in Table 4 , $\underline{v}_{r e I}$ is of the order of $10 \mathrm{~m} / \mathrm{s}$. For these scenarlos the longltudinal wind component will be of limfted value In clearling the beam in the vicintty of the stagnation point.

Table 4. Residence time in beam for atr particles passing through stagnation point.

\begin{tabular}{lccc} 
Scenario & $\begin{array}{c}\text { Target position } \\
\text { at } \begin{array}{c}t=0 \\
(\mathrm{~km})\end{array}\end{array}$ & $\begin{array}{c}\text { Stagnation point } \\
\text { at } t=0 \\
(\mathrm{~km})\end{array}$ & $\begin{array}{c}\text { Residence time, } \\
\tau \\
(\mathrm{s})\end{array}$ \\
\hline A & 1.5 & 0.844 & 1.0 \\
B & 1.0 & 0.379 & 0.5 \\
C & 2.5 & 2.33 & 1.7 \\
D & 1.0 & 0.295 & 1.0 \\
\hline
\end{tabular}



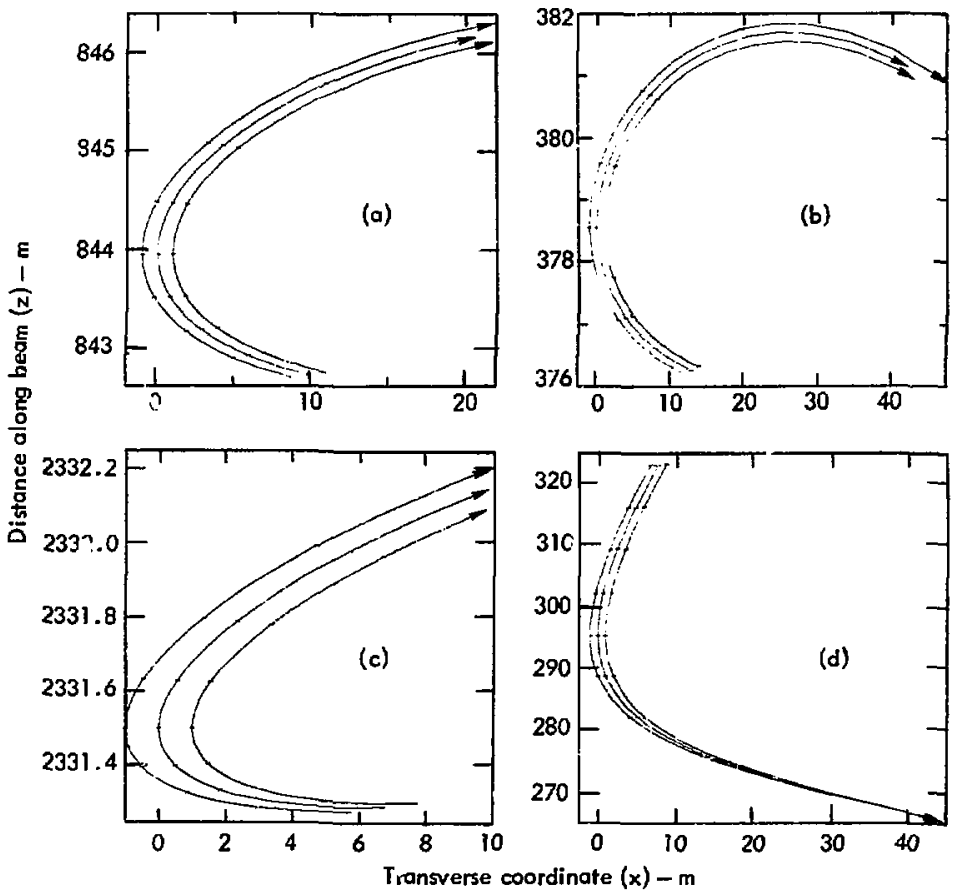

Fig. 10 . A1r-flow trajectories in rest frame of slewing laser beam in the presence of a stagnation zone. Ticks on the curves denote $0.5-s$ intervals. (a) Range $=1.5 \mathrm{~km}$, stagnation point at $z=0.844 \mathrm{~km}$. (b) Range $=1.0 \mathrm{~km}$, stagnation point at $z=0.379 \mathrm{~km}$. (c) Kange $=2.5 \mathrm{~km}$, stagnation point at $z=2.33 \mathrm{~km}$. (d) Range $=1 \mathrm{~km}$, stagnation point at $z=0.295 \mathrm{~km}$.

\section{Calculation of Transverse Wind Velocities for Noncoplanar Scenarios}

We shal: again assume the Bcenario of $\mathrm{FIg}$. 1 , only now we shaIl relax the, assumption that the scenarlo or kinematlc plane necessarlly colncides with the earth's, or the horlzontal, plane. The line PLP and the wind vector, however, w111 be assumed to lie in the earth's plane (see Fig. 11), Again, the $x$ direction will be along the direction 


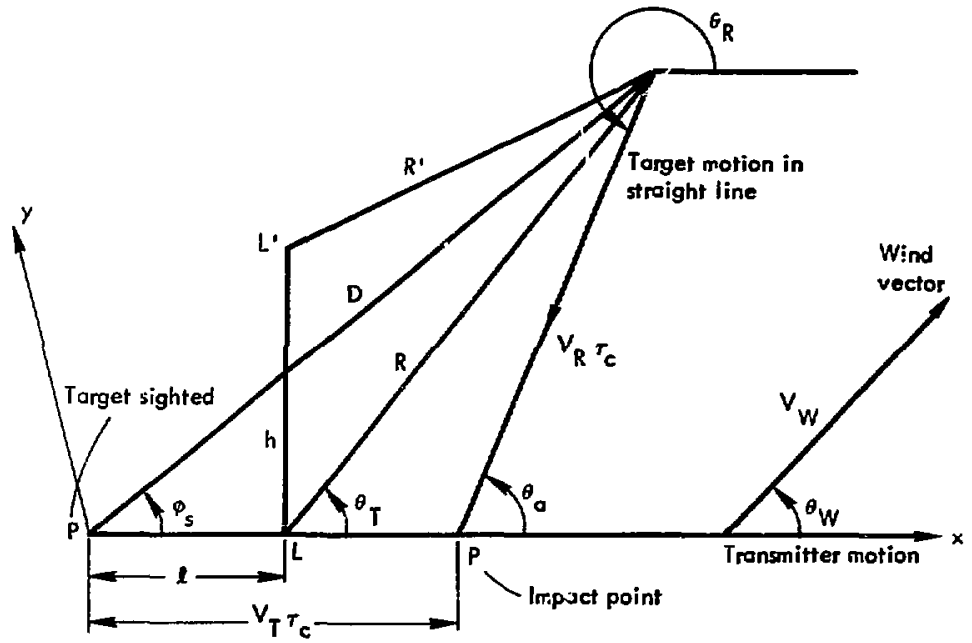

Fig. 11. Diagram of noncoplanar scenario. Laser is now sltuated at a height $h$ above the platform.

of motion of the laser platform, the $y$ direction will be in the kinenatic plane, and the unit vector normal to the kinematic plane will be called $\hat{\zeta}$. The laser aperture will be situated at position $L^{\prime}$, which is at a height $h$ aboy the line PLP. The line $L L$ ' defines the vector $\underline{h}=$ $\underline{h}=h \hat{h}$, which is normal to the horizontal plane and makes an angle $\theta_{p}$ with the vector $\hat{5}$. The scenario parameters $D_{s} \phi_{s}, R, \theta_{R}, Z$, and $\theta_{T}$ are now defined in a plape tilted with respect to the horizontal plane, but they are related exactly the same as before. The distance $R$, however, no longer has the significance of range. The calculation of the true range $R^{\prime}$ is described below.

In order to follow the wind in a frame of reference that moves with the laser, it is necessary to introduce an approprtate orthogonal coordinate system. Clearly this coordinate system will not be unique, but a suitable one san be defined as follows: 2 is directed along the laser beam,

$$
\hat{y}^{\prime}=\frac{\hat{z} \times \underline{\mathrm{V}}_{T}}{\left|\hat{\mathrm{z} \times \mathrm{V}_{-}}\right|},
$$

and

$$
\hat{x}^{\prime}=\hat{y}^{\prime} \times \hat{z} .
$$

It is most convenient to express all vectors used in the computation in 
the kinematic coordinate system.

Hence we have

$$
\begin{aligned}
& \hat{V}_{T}=(1,0,0), \\
& \hat{V}_{R}=\left(\cos \theta_{R}, \sin \theta_{R}, 0\right), \\
& \hat{R}=\left(\cos \theta_{T}, \sin \theta_{T}, 0\right), \\
& \hat{h}=\left(0, \sin \theta_{p}, \cos \theta_{p}\right), \\
& \hat{V}_{W}=\left(\cos \theta_{W}, \sin \theta_{W} \cos \theta_{p},\right. \\
& \left.-\sin \theta_{W} \sin \theta_{p}\right)
\end{aligned}
$$

$\underline{\mathbf{R}}^{\prime}=\underline{\mathbf{R}}-\underline{\mathbf{h}}$,

(12a) where $\hat{V}_{T}, \hat{V}_{R}, \hat{V}_{W}$ are unit vectors directed along $\underline{v}_{T}, \underline{\nabla}_{R}$, and $\underline{v}_{W}$, and the vectors $\underline{R}=R R$ and $\underline{R}^{\prime}$ are directed along lines excending from $L$ and $L^{\prime}$, respectively, to the receiver (target).

The effective wind seen in the frame of reference moving with the laser beam is

$$
\underline{\mathrm{V}}_{\mathrm{eff}}(z, t)=\left(\underline{\nabla}_{W}-\underline{\mathrm{V}}_{T}\right)-\frac{z}{R^{\prime}}\left\{\left[\underline{\nabla}_{R}-\left(\underline{\mathrm{V}}_{\mathrm{i \textrm {i }}}{ }^{*} \hat{\mathrm{s}}\right) \hat{z}\right]-\left[\underline{V}_{T}-\left(\underline{\mathrm{V}}_{T} \cdot \hat{z}\right) \hat{z}\right]\right\} .
$$

The effective wind components along $\hat{z}^{\prime}$ and $\hat{y}^{\prime}$ are then obtained from

$$
\begin{aligned}
& V_{\text {eff } x^{\prime}}=\underline{v}_{\text {eff }} \cdot \hat{x}^{\prime}, \\
& V_{\text {eff } y^{\prime}}=\underline{v}_{\text {eff }} \cdot \hat{y}^{\prime} .
\end{aligned}
$$

The effective horizontal and vertical wind components in Eqs. (14) become inputs to the hydrodynamic calculation which is described in Sections 6 and 7 .

\section{Steady-State Solutions of Hydrodynamic Equations for Arbitrary Transverse Wind Velocities: cw Steady State}

Noncoplanar scenarlos create effective winds whose orientation in the transverse plane vary with propagation distance a. All symmetry in the transverse plane is lost, and the $x$ axis can no longer serve as the wind axis. The linearized hydrodynamic equations must be recast and solved for a wind having an arbitrary direction.
The linearized hydrodynami: equations to be solved are

$$
\begin{gathered}
\frac{\mathrm{d} \rho_{1}}{\mathrm{~d} t}+\rho_{0} \underline{\nabla} \quad \underline{v}_{1}=0 \\
\rho_{0} \frac{\mathrm{d}}{\mathrm{d} t} \underline{v}_{1}=-\underline{\nabla}_{1}+\hat{e}_{i}-\frac{\partial}{\partial x_{j}}\left[\eta \left(\frac{\partial v_{1 i}}{\partial x_{j}}\right.\right. \\
\left.\left.+\frac{\partial v_{1 j}}{\partial x_{i}}-\frac{2}{3} \delta_{i j}-\underline{v}_{-1}\right)\right] \\
\frac{\mathrm{d}}{\mathrm{d} t}\left(p_{1}-c_{s}{ }^{2} \rho_{1}\right)=(r-1) \alpha I,
\end{gathered}
$$


where $\rho_{1}, \underline{v}_{1}$, and $p_{1}$ represent the density, velocity, and pressure perturbations induced by laser heating, $\eta$ is the viscosity, and the total derivative $\frac{d}{d t}$ is defined by

$$
\frac{\mathrm{d}}{\mathrm{d} t}=\frac{\partial}{\partial t}+v_{x} \frac{\partial}{\partial x}+v_{y} \frac{\partial}{\partial y}
$$

Elimination of $p_{1}$ and $v_{1}$ ylelds the fallowing equation for $p_{1}$ :

$$
\begin{gathered}
\frac{d}{d t}\left(\frac{d^{2}}{d t^{2}} \rho_{1}-c_{s}{ }^{2} \nabla^{2} \rho_{1}-\frac{4}{3} \frac{\pi}{\rho_{0}} \nabla^{2} \rho_{1}\right) \\
=(r-1) a \nabla^{2} I .
\end{gathered}
$$

We are Interested in the steady state or the case in which Eq. (17) becomes

$\left[\left(v_{x} \frac{\partial}{\partial x}+v_{y} \frac{\partial}{\partial y}\right)^{2}-c_{\varepsilon}^{2} \nabla^{2}-\frac{4}{3} \frac{\eta}{\rho_{0}} \nabla^{2}\left(v_{x} \frac{\partial}{\partial x}+v_{y} \frac{\partial}{\partial y}\right)\right] \dot{\rho}_{1}=(\gamma-1) \alpha \nabla^{2} I$,

where

$$
\dot{\rho}_{1}=v_{x} \frac{\partial \rho_{1}}{\partial x}+v_{y} \frac{\partial \rho_{1}}{\partial y} .
$$

The solution for $\rho_{1}$ is carried out in two steps: flrst Eq. (18) is solved with $\dot{\rho}_{1}$ as dependent variable, and then Eq. (19) is solved for $\rho_{1}$.

We shall restrict our attention to the subsonic case, where $v_{x}^{2}+v_{y}^{2}$ $<c_{B}^{2}$. In that case Eq. (1B) Is ellip- tic and can be expressed in terms of a finite Fourler series repregentation:

$\dot{\rho}_{1}(x, y)$

$$
=\sum_{k_{x}, k_{y}} \tilde{\dot{\rho}}_{1}\left(k_{x}, k_{y}\right) \exp \left[i\left(k_{x} x+k_{y} y\right)\right] .
$$

$$
\dot{\tilde{\rho}}_{1}\left(k_{x}, k_{y}\right)=-\frac{(\gamma-1)}{c_{s}^{2}} \frac{\alpha \widetilde{T}\left(k_{x}, k_{y}\right)\left(k_{x}^{2}+k_{y}^{2}\right)}{\left\{\left(k_{x}^{2}+k_{y}^{2}\right)\left[I+\frac{4}{3} i \frac{\eta}{\rho_{0} e_{z}^{2}}\left(v_{x} k_{x}+v_{y} k_{y}\right)\right]-\frac{\left(v_{x} k_{x}+v_{y} k_{y}\right)^{2}}{c_{s}^{2}}\right\}},
$$

where $\tilde{I}\left(k_{x}, k_{y}\right)$ is the Fourler transform of $I(x, y)$. The inverse Fourter transform of Eq. (21) Is evaluated by means of the fast Fourler transform (FFT) algorithm. The function $\dot{p}_{1}(x, y)$ thus obtalned then becomes 
the source term for $\mathrm{Eq}$. (19), which can be solved using the Carlson method ${ }^{1}$ for integration along characteristics. The solution of Eq. (19) is obtained by a difference method in configuration space in preference to a Fourier transform method because the transform of $\rho_{1}(x, y)$ will have poles whenever $v_{x} k_{x}+v_{y} k_{y}=0$. The evaluation of the Inverse cransform by the FFT algorithm will be troublesome, since these poles must be avoirled.
If we define

$$
\begin{aligned}
\beta & =\left|\frac{v_{y} \Delta_{x}}{v_{x} \Delta_{y}}\right|, \\
i^{\prime} & =\frac{v_{x}}{\left|v_{x}\right|}, \\
j^{\prime} & =\frac{v_{y}}{\left|v_{y}\right|},
\end{aligned}
$$

the difference equation satisfled by $\rho_{i j}=\rho_{1}(i \Delta x, j \Delta y)$ can be written

$$
\begin{aligned}
& \rho_{i, j}=\frac{1}{\beta} \rho_{i-i, j, j}+\left(1-\frac{1}{\beta}\right) \rho_{i, j-j^{\prime}} \\
& +\frac{\Delta_{y}}{2\left|\nu_{y}\right|}\left\{\dot{\rho}_{i, j}+\frac{1}{\beta} \dot{\rho}_{i-i^{\prime}, j-j^{\prime}}+\left(1-\frac{1}{\beta}\right) \dot{\rho}_{i, j-j^{\prime}}\right\} \text { for } \beta>1 \text {; } \\
& \rho_{i j}=\beta \rho_{i-i^{\prime}, j^{\prime} j^{+}}+(1-\beta) \rho_{i-i^{\prime}, j} \\
& +\frac{\Delta}{2\left|v_{x}\right|}\left\{\dot{\rho}_{i j}+e \dot{\rho}_{i-i^{\prime}, j^{\prime}-j^{\prime}}+(1-\beta) \dot{\rho}_{i-i^{\prime}, j}\right\} \text { for } B \leq 1
\end{aligned}
$$

\section{Steady-State Solutions of Hydrodynamic Equations for Arbitrary Transverse Wind Velocities: Multipulse Steady State}

Isobaric density changes induced by multipulse heating are governed by the equation

$$
\frac{\partial \rho_{1}^{\mathrm{mp}}}{\partial t}+v_{x} \frac{\partial \rho_{1}^{\mathrm{mp}}}{\partial x}+v_{y} \frac{\partial \rho_{1}^{\mathrm{mp}}}{\partial y}=-\frac{\gamma-1}{c_{B}^{2}} \alpha \sum_{n} \tau_{i} T_{n}(x, y) \delta\left(t-t_{n}\right) .
$$

where $\left.\tau_{p} I_{n} ; x, y\right)$ represents the fluence of the $n$th pulse, and $\tau_{p}$ represents 
the pulge width. If "steady-state" conditions prevail, it can be assumed that $I_{n}$ does not vary from pulse to pulse. Hence $I_{n}(x, y)=I(x, y)$. Taking the Fourler transform of Eq. (24) with respect to $x$ and $y$ yields

$$
\frac{\partial \tilde{\rho}_{1}^{m p}}{\partial t}+i\left(v_{x} k_{x}+v_{y} k_{y}\right) \tilde{\rho}_{1}^{-m p}=-\frac{Y-1}{c_{s}^{2}} \alpha \tau_{p} \tilde{I}\left(k_{x}, k_{y}\right) \sum_{n} \delta\left(t-t_{n}\right) .
$$

Solving Eq. (25) for $\tilde{\rho}_{1}^{m p}$ at a tıme $t=m \Delta t$, where $m$ 1s any integer and $\Delta t$ is the time Interval between successive pulses, gives

$$
\tilde{\rho}_{1}\left(k_{x}, k_{y}, m \Delta t\right)=-\frac{Y-1}{c_{s}^{2}} \alpha \tilde{I}\left(k_{x}, k_{y}\right) \sum_{n=1}^{N_{p}} \exp \left[-i n \Delta t\left(k_{x} v_{x}+k_{y} v_{y}\right)\right] .
$$

The exponentials in Eq. (26) correspond to translations of the individualpulse fluence distributions in configuration space by wind motion.

The summation begins with $n=1$ because the 1sobaric density changes created by a given pulse do not have time to develop during the pulse width, $\tau_{p}$. The upper limit $N_{p}$ is bused on numerical considerations and is determined by

$$
\begin{gathered}
N_{P}=\operatorname{MIN}\left(N_{\text {input }},\left[\frac{N \Delta x}{\left|v_{x}\right| \Delta t}\right],\right. \\
\left.\left[\frac{N \Delta y}{\left|v_{y}\right| \Delta t}\right]\right),
\end{gathered}
$$

where $N$ is the numerical length of the mesh used for solving the wave equation.
In Eq. (27) MIN signifies the minImum of the arguments, and the square brackets represent the integer part of the arguments inside them. An input value of $N_{p}$ is iseful if a true stagnation point is encountered along the propagation path. In such cases the total density change at the stagnation point can be kept bounded. For example, $N_{\text {input }}$ might be set equal to the actual number of pulses in a given train, in whick case a true lower bound could be assigned to the intensities at the target.

The remaining arguments in $\mathrm{Eq}$. (27) prevent any pulse fluence distribution from affecting the density calculation If It has been translated by more than the minfmum (physical) dimension of the computational mesh for the wave equation, 1.e. MIN $(N \Delta x, N \Delta y)$. 
The density calculation itself is carried out on a $2 N \times 2 N$ mesh; which has a buffer of length $N$ in both the $x$ and $y$ directions. Thus if $N_{P}$ satisfles condition (27), perlodic "wrap-around" or positional aliasing of the densty contributions by past pulses in the train is avolded.

The summation in Eq. (26) may be evaluated directly, and $\tilde{D}_{1}$ can be expressed in the form

$$
\begin{array}{r}
\tilde{p}_{1}\left(k_{x}, k_{y}\right)=-\frac{\gamma-i}{c_{s}^{2}} \alpha \tilde{T}\left(k_{x}, k_{y}\right) \exp \left[-i \frac{n_{p}+1}{2} \Delta t\left(k_{x} y_{x}+k_{y} y_{y}\right)\right] \\
\times \frac{\sin \frac{N_{p}}{2} \Delta t\left(k_{x} y_{x}+k_{y} y_{y}\right)}{\sin \frac{\Delta t}{2}\left(k_{x} v_{x}+k_{y} v_{y}\right)} .
\end{array}
$$

The density $\rho_{1}(x, y)$ is than obtalnable from Eq. (28) by an trverse transform operation using the FFT algorithm. Equation (28) has been used in a number of test examples with satisfactory results. If the spectrum $I\left(k_{x} k_{y}\right)$ is particularly rich in high spatial frequencles, Eq. (28) may give rise to a ringing behavior in configuration space due to the fact that the shift operators $\exp \left(-i n \Delta t k_{x} v_{x}\right), \exp \left(-i n \Delta t k_{y} v_{y}\right)$ may not correspond to lattice translation operators on the cociputacional mesh. In ruch a case ringing can be suppressed by expressIng the solution of Eq. (26) in terms of the interpolations of lattic: shift operations,

By means of bilinear interpolation, one can express any function $T(x, y)$ at positions intermediate to the lattice by means of

$$
\begin{aligned}
T_{j+f_{x}, k+f_{y}}=\left(1-f_{y}\right) f_{x} T_{j+1, k} & +\left(1-f_{x}\right) f_{y}{ }^{T}{ }_{j, k+1} \\
& +f_{x} f_{y} T_{j+1, k+1}+\left(1-f_{y}\right)\left(1-f_{x}\right) T_{j, k} .
\end{aligned}
$$

$0<f_{x} \leq 1,0<f_{y} \leq 1$, 
where $f_{x}$ and $f_{y}$ reprasere fractlonal distances between lattice coordinates, and where the numbers $\tau_{j}$, represent values of $t(x, y)$ sampled at lattice points. The sumarion in Eq. (26) my thus be represenced in the following alcernative form, which avolds che use of nonlectice shlift operators (the notation ( ) signifles the integer part of the argument):

$$
\begin{aligned}
& \text { Hit } \\
& \sum_{x=1} \exp 1-i n h t\left(i_{x} y_{x}+i_{y} y_{y}\right) j \\
& -\sum_{n=1}^{n}\left\{f _ { x } ( 1 - j _ { y } ) \operatorname { e x p } \left(i \frac{\pi}{n}\left\{n_{i_{x}}\left(\left[n_{x} n\right)+1\right)+n_{k_{y}}\left(n_{y} n \mid\right\}\right)\right.\right. \\
& \left.+i_{y}\left(1-i_{y}\right) \operatorname{axp}\left(i \frac{\pi}{\pi}\left\{n_{k_{x}}\left[n_{x} n\right)+n_{k_{y}}\left(1 n_{y} n\right]+1\right)\right\}\right) \\
& +r_{y} \exp \left(i \frac{\pi}{i}\left\{n_{k_{x}}\left(\left[n_{x} n\right]+1\right)+n_{k_{y}}\left(\left[n_{y} n\right]+1\right)\right\}\right) \\
& \left.+\left(1-f_{y}\right)\left(1-f_{x}\right) \exp \left(i \frac{\pi}{n}\left\{n_{k_{x}}\left[n_{x} n\right]+n_{y_{y}}\left[n_{y} n\right]\right\}\right)\right\}-g\left(k_{x}, k_{y}\right) \text {, }
\end{aligned}
$$

where

$$
\left.\begin{array}{rl}
\eta_{x} & =\frac{\nu_{x}^{\Delta t}}{\Delta x}, \\
\eta_{y} & =\frac{\nu_{y} \Delta t}{\Delta y}, \\
f_{x}(n) & =\eta_{x} n-\left[\eta_{x} n\right], \\
f_{y}(n) & =\eta_{y} n-\left[\eta_{y} n\right] .
\end{array}\right\}
$$

The summation in Eq. (26) over $n$ can be evaluated as $a ? N \times 2, N$ DFT with the aid of the FFI algorithm. For a Given value of $n$, the numbers $\left[n_{x} n\right]$, $\left[n_{x} n\right]+1$ can each be identified as $x$-coordinates $n_{x}$ and the numbers $\left[n_{y} n\right],\left[m_{y} n\right]+1$ as $y$-coordinates $n_{y}$ In the lattice space. Thus each exponential in the summation in Eq. (31) can be identified with a parcicular latelce point $n_{x}, n_{y^{*}}$ As the lindex $n$ ls incremented, the approprtace blsinear function of $f_{x}(n)$ and 
$\hat{f}_{y}(n)$ is added to the contents of a storage reglster corresponding to coordinates $n_{x}, n_{y}$, On completion of this operation, a two-dimensional DFT of the resultant array wil yield the desfred sum (31).

The Fourfer transform of the density can then be expressed as

$$
\begin{gathered}
\tilde{\rho}_{1}\left(k_{x}, k_{y}, m \Delta t\right)=-\frac{(\gamma-1)}{c_{s}^{2}} \\
\times \alpha \tilde{I}\left(k_{x}, k_{y}\right) \tilde{g}\left(k_{x}, k_{y}\right) .
\end{gathered}
$$

Both the options (28) and (29) are currently avallable in the Four-D code, and the cases run have produced results that are almost indistinguishable.

The shifts and Interpolations Implied in Eq. (31) may, of course, be carrled out strictly in configuration space. If $v_{p}$ is small, this procedure may be more economica!. As $\tilde{r}_{i}$, becomes large, the Fourier trans form method becomes more economical.

\section{Effect of Noncoplanarity on Propagation of cw Laser Beams Through Stagnation Zones}

We shall focus attention on the scenario discussed In Ref. 1 , in which the total propagation distance is $1.5 \mathrm{~km}$ and the stagnation point occurs at $a=0.8439 \mathrm{~km}$. The initial diffraction-limited beam is Gaussian, with $1 / e^{2}$-intensity diameter of $70 \mathrm{~cm}$, and is assumed to be focused at the $1.5-\mathrm{km}$ range. The wavelength and absorption coefficlent are assumed to be $3.8 \mu \mathrm{m}$ and $0.07 \mathrm{~km}^{-1}$, respectively. For reference the results of the time dependent calculations at $t=60 \mathrm{~ms}$ are given in Table 5. For this value of $t$, the beam properties are changing very slowly, and the assump- tion of a "quasl" steady state is a reasonable one.

In the noncoplanar scenario, on the other hand, a true steady state Is known to exist, and a time to establish this steady state can be estimated by dividing the bean dlameter by the magnitude of the vertical wind component at the stagnation point. The noncoplanar results are naturally much cheaper to obtain than the corresponding coplanar results.

In Table 6 , steady-state results are given for the scenario corresponding to Table 5 for a variety of elevations of the laser aperture 
Table 5. Heam properties on target at $t=60$ ms.

\begin{tabular}{|c|c|c|c|}
\hline $\begin{array}{l}\text { Laser power } \\
\text { (kW) }\end{array}$ & $\begin{array}{l}\text { Peak Intensity } \\
\text { at target } \\
\left(\mathrm{kW} / \mathrm{cm}^{2}\right)\end{array}$ & $\begin{array}{l}\text { Mintmum half- } \\
\text { power area } \\
\left(\mathrm{cm}^{2}\right)\end{array}$ & $\begin{array}{c}\text { Intensity averaged } \\
\text { over oinimum } \\
\text { half-power area } \\
\text { (kw/ } / \mathrm{cm}^{2} \text { ) }\end{array}$ \\
\hline 500 & 10.8 & 33.4 & 6.72 \\
\hline $500^{a}$ & 9.8 & 53.5 & 4.19 \\
\hline $500^{b}$ & 11.0 & 33.2 & 6.76 \\
\hline 250 & 12.4 & 13.5 & B. 34 \\
\hline 125 & 17.7 & 4.42 & 12.7 \\
\hline 62.5 & 22.7 & 1.65 & 17.0 \\
\hline
\end{tabular}

${ }^{\mathrm{a}}$ Focus $100 \mathrm{~m}$ beyond range.

botion of stagnation zone taken into account.

Table 6. Steady-state cw beam properties as a function of laser height above scenario plane.

\begin{tabular}{|c|c|c|c|c|c|c|c|}
\hline $\begin{array}{l}\text { Laser } \\
\text { power } \\
\text { (kW) }\end{array}$ & $\begin{array}{l}\text { Laser } \\
\text { elevat1on } \\
\text { (m) }\end{array}$ & $\begin{array}{l}\text { Vertical wind } \\
\text { speed at } \\
\text { stagnation point } \\
\text { (m/g) }\end{array}$ & $\begin{array}{l}\text { Minimim half- } \\
\text { power area } \\
\text { (osagnation } \\
\text { point) } \\
\text { (cm })\end{array}$ & $\begin{array}{l}\text { Minlmum half- } \\
\text { pourer area } \\
\text { (target) } \\
\left.\text { (cm }{ }^{2}\right)\end{array}$ & $\begin{array}{l}\text { Tige to } \\
\text { oteady } \\
\text { otate } \\
\text { (B) }\end{array}$ & $\begin{array}{l}\text { Peak } \\
\text { Intensity } \\
\text { at target } \\
\left(\mathrm{kW} / \mathrm{cm}^{2}\right)\end{array}$ & $\begin{array}{l}\text { Intengl ty } \\
\text { averaged over } \\
\text { ofnimum half- } \\
\text { power area at } \\
\text { target } \\
\text { (kW/on }{ }^{2} \text { ) }\end{array}$ \\
\hline \multirow[t]{5}{*}{500} & 5 & 0.55 & 293 & 37.7 & 0.312 & 11.9 & 5.97 \\
\hline & 10 & 1.1 & 291 & 33.6 & .155 & 12.0 & 6.69 \\
\hline & 20 & 2.2 & 290 & 29.1 & .077 & 14.0 & 7.72 \\
\hline & 30 & 3.3 & 289 & 26.4 & .052 & 15.9 & 6.52 \\
\hline & 40 & 4.4 & 287 & 23.6 & .039 & 16.1 & 9.53 \\
\hline \multirow[t]{5}{*}{250} & 5 & 0.55 & 279 & 13.7 & .303 & 16.6 & 8.2 \\
\hline & 10 & 1.1 & 278 & 12.0 & .152 & 17,8 & 9.36 \\
\hline & 20 & 2.2 & 277 & 10.1 & .076 & 19.5 & 11.0 \\
\hline & 30 & 3.3 & 278 & 8.9 & .051 & 21.6 & 12.6 \\
\hline & 40 & 4.4 & 276 & 7.88 & .038 & 24,1 & 14.3 \\
\hline \multirow[t]{4}{*}{125} & 5 & 0.55 & 272 & 4.66 & .300 & 22.9 & 12.0 \\
\hline & 10 & 1.1 & 271 & 4.04 & .150 & 25.3 & 13.9 \\
\hline & 20 & 2.2 & 271 & 3.33 & .075 & 28.6 & 19.5 \\
\hline & 40 & 4.4 & 270 & 2.31 & .037 & 39.: & 24.3 \\
\hline \multirow[t]{2}{*}{62.5} & 5 & 0.55 & 268 & 1.58 & .29 & 30.6 & 17.7 \\
\hline & 10 & 1.1 & 268 & 1.37 & 0.14 & 34.3 & 20.5 \\
\hline
\end{tabular}



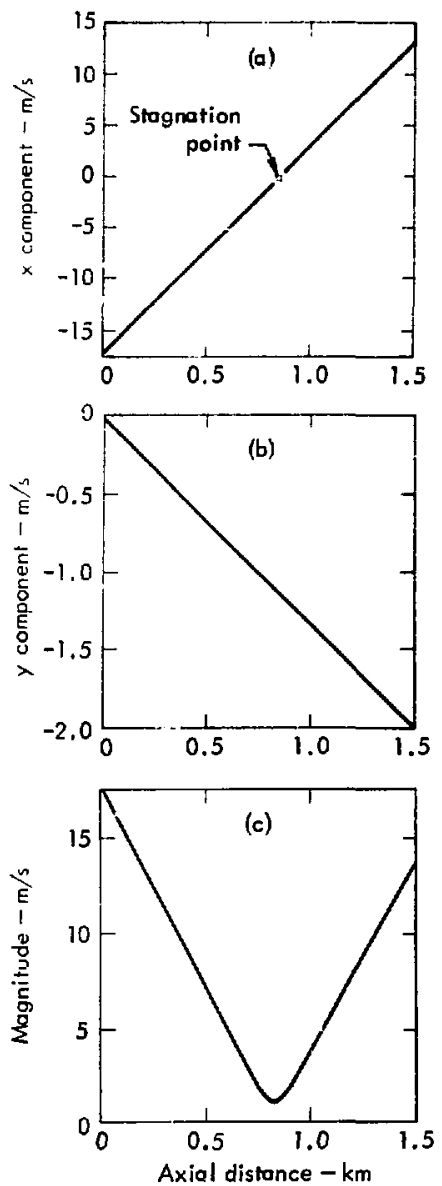

Fig. 12, Transverse wind veloctty as a function of axia1 distance for cw beam. (a) $x$ component. (b) y component. (c) Magnitude. above the scenario plane. Figure 12 shows the variations with $a$ of the horizontal and vertical components and the magnitude of the transverse wind.

From Tables 5 and 6, it is evident that the space-aseraged Intengities if the focal plane for the noncoplanar scenario at 5-m elevation agree with the corresponding average steady-state intensities for

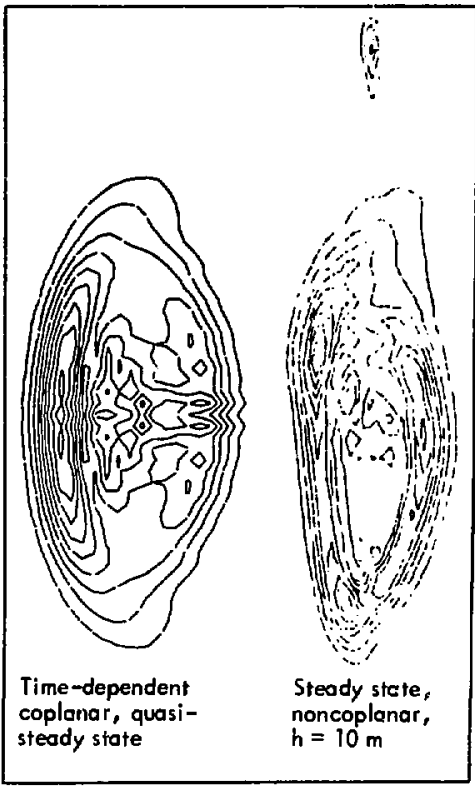

F1g. 13. Comparison of isointensity contours for stagnationzone situations in coplanar and noncoplanar cases. 
the coplariar scenartos to within less than $10 \%$. The peak intensities for the noncoplanar scenario at $5 \mathrm{~m}$, on the other hand, are somewhat higher than the corresponding values for the coplanar case. There is also a substantial difference in the appearance of the isoinienstty concours in the focal plane ( $\left.f I_{g}, 13\right)$. As would be expected, performance Improves with height, although the improvement is marginal for the elevations considered. In all cases a steady-state condition can be reached in a time small compared with times of interest.

In conclusion, average intens 1ties for coplanac stagnation-zone scenarlos can be calculated by adding nominal noncoplanar features to the scenar10 and performing a steady-state calculation. For iw beams, however, rather substantial Jaser elevations musc be provided co alleviate stagnation-zone effects.

\section{Effect of Noncoplanarity of Propagation of Multipulge Beams Through Stagnation Zoneg}

We turn our attention again to the scenario of Section 3. All problem parameters are the same, except that the laser is now assumed to be elevated $10 \mathrm{~m}$ above the scenario plane. Figure 14 shows the vertical and horizontal components of transverse wind velocity as functions of propagation distance. Figure 15 shows the Isointensity contours in the target plane for the various repetition rates.

Table 7 compares laser performance on target as a function of pulse-repetition frequency for the coplanar scenarto and the noncoplanar gcenarto with a laser elevation of $10 \mathrm{~m}$. In the absence of complete steady-state data for the coplanar case, we have used in Table 7 intensity values corresponding to the final times exhibited in Fig. 6 for a given value of $v$. Thus the improvements due to noncoplanarity shown In Table 7 are conservative estimates.

It Is seen from Table 7 that Improvements of at least a factor of 2 , conservatively estimated, are possible for all values of $v$. In the case of $v=10 \mathrm{~s}^{-1}$ the laser performance is even better than it would be in a vacuum. The reason is that for this pulse-repetition frequency the overlap number at the stagnation point is on $1 y 2$, and for 

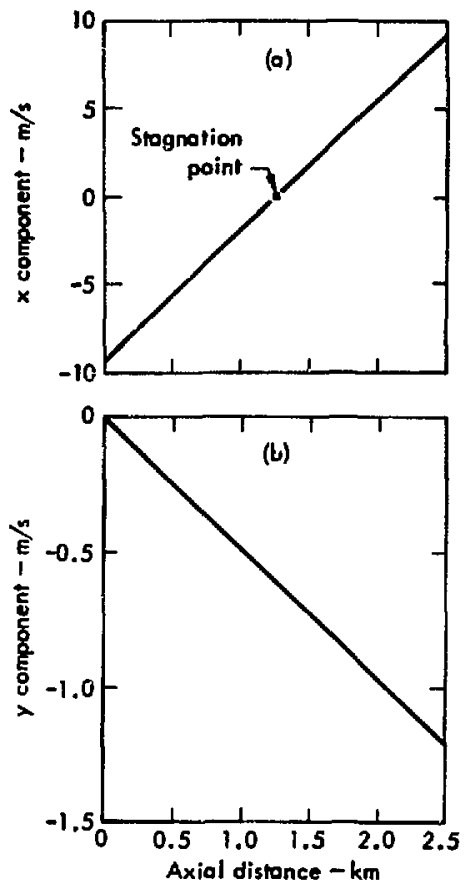

F1g. 14. Transverse wind velocity as a iunction of axial distance for multipulse beam. (a) $x$ component. (b) y component.

overlap numbers in the range 1-?

such enhancement effects for multipulse beams are well known. 6

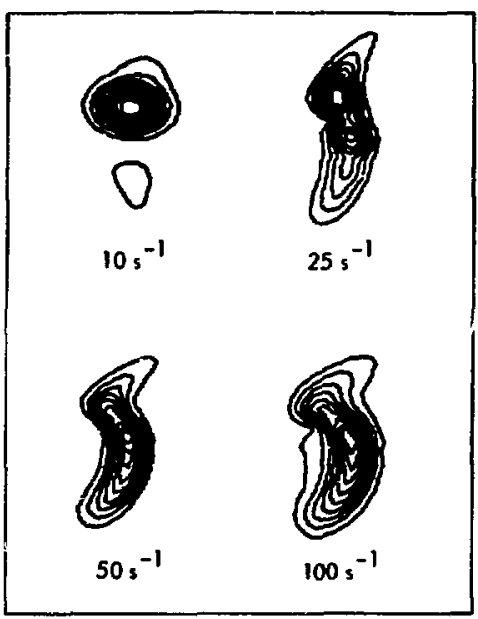

Fig. 15. Changlng shapes of isolntensity contours as a function of pulse-repecticion rate for noncoplanar scenario; laser at $10-\mathrm{m}$ elevation.
To summarize: there is clearly some hope of mininizing stagnationzone blooming for multipulse beams by a combination of elevating the laser aperture above the scenario plane and lowering the pulserepetition frequency. 
Table 7. Comparison of multipluse beam properties for coplanar and noncoplanar scenarios. Power $=53 \mathrm{kH}$, range $n 2.5 \mathrm{~km}, \lambda=10.6 \mathrm{~km}$, elevation $h=10 \mathrm{~m}$, and vertical wind speed at stagnation point $=0.61 \mathrm{~m} / \mathrm{s}$.

\begin{tabular}{|c|c|c|c|c|c|c|c|}
\hline $\begin{array}{l}\text { Pulse } \\
\text { repetition } \\
\text { frequency, } \\
\text { (s-1) }\end{array}$ & $\begin{array}{c}\text { MInlmur } \\
\text { half-power } \\
\text { area } \\
\text { (otagnation } \\
\text { polnt, } \\
\text { noncoplanar } \\
\text { scenaylo) } \\
\left(c_{a}^{-2}\right)\end{array}$ & $\begin{array}{l}\text { Tine to } \\
\text { aready } \\
\text { gtake } \\
\text { (non- } \\
\text { coplenar } \\
\text { sconarto) } \\
\text { (s) }\end{array}$ & $\begin{array}{l}\text { Ovirlap } \\
\text { nubber ac } \\
\text { gragnetion } \\
\text { polnt (non- } \\
\text { coplanar } \\
\text { scenario) }\end{array}$ & $\begin{array}{c}\text { Peak } \\
\text { incengity } \\
\text { at tarbet } \\
\text { (coplanar } \\
\text { scenario) } \\
(\text { (w/ch } 2)\end{array}$ & $\begin{array}{c}\text { Pesk } \\
\text { Incensity } \\
\text { at carget } \\
\text { (nan- } \\
\text { coplanar } \\
\text { acentiolo) } \\
\text { (H/en 2) }\end{array}$ & $\begin{array}{c}\text { Intensity } \\
\text { overased } \\
\text { over } \\
\text { minima } \\
\text { helf-pover } \\
\text { area } \\
\text { (coplanar } \\
\text { gcenario) } \\
\text { (H/cm 2) }\end{array}$ & 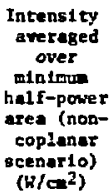 \\
\hline 10 & 131 & 0.19 & 1.9 & $85.5^{\mathrm{c}}$ & $287^{\circ}$ & $52.0^{\circ}$ & $181^{b}$ \\
\hline 25 & 116 & 0.18 & 4.49 & $: 0.5^{c}$ & 116 & $32.5^{c}$ & 65.6 \\
\hline 50 & 104 & 0.17 & 8.49 & $28.7^{\mathrm{d}}$ & 70.9 & $17.8^{d}$ & 42.3 \\
\hline 100 & 140 & 0.19 & 19.0 & $30.4^{d}$ & 49.0 & $13.2^{\mathrm{e}}$ & 30.3 \\
\hline
\end{tabular}

\footnotetext{
Qvacuum beam has value 238.

baxum beam has value 170 .

c. $0.6 \mathrm{~s}$, uteacy state has not been rewerhed.

$d_{t}=0.32 \mathrm{~s}$, sceady state has not been reached.

': $=0.2 \mathrm{~s}$, steady state has not been reached.
}

\section{Single-Pulse Thermal Blooming in the Triangular Pulse Approximation}

The Isobaric approximation for changes in air density is invalid for a single laser pulse whose duration is comparable to or less than the transit time of sound across the beam. In this time regime - referred to as the $t^{3}$-regime because of the time dependence of density changes arising from an applied constant laser-energy absorption rate - the air-density changes must be determined from the complete set of timedependent hydrodynamic equations, Eqs. (15).1,7
At late times in the pulse, $t^{3}$ thermal blooming tends to reduce the on-axis intensity relative to what It would be if the beam were propagating in vacuum. This reduction increases with time, and for sufficiently late times a depression appears in the center of the beam. Energy added to the pulse at later times will contribute only marginally to the on-axis fluence. Thus, for a spectfic peak pulse intensity, the on-axis fluence appears to saturate as the pulse 
duration is stretched out more

and more.

These properties are best illustrated by a numerical example. Let us consider a beam that is Gaussian at $z=0$ with $1 / e^{2}$-intensity radius $25 \mathrm{cr}$. The beam, which is focused at $2,2 \mathrm{~km}$, is assumed to be $2 \times$ diffraction Iimited ( $\lambda$-scaled) with $\lambda=10.59 \mu \mathrm{m}$ and $\alpha=0.3 \times 10^{-5} \mathrm{~cm}^{-1}$. The pulse is souare-shaped in time and lasts $100 \mu \mathrm{s}$. The choice of a square-shaped pulse is convenient because a single calculation contains the complete information for all square pulses of duration shorter than the one chosen.

FIgure 16 shows the on-axis intensity at $z=2.0 \mathrm{~km}$, obcained

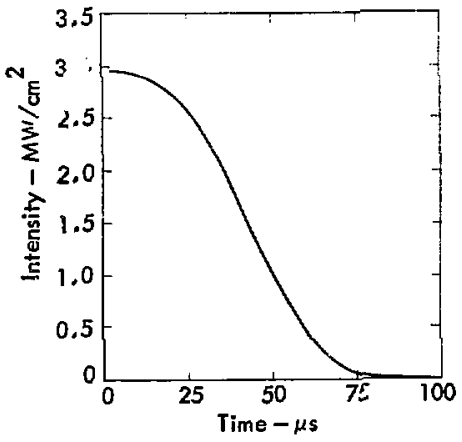

Fig. 16. On-axis intensity as 1 function of time. The pulse Is taken to be square-shaped in time. Thermal blooming reduces on-axis intensity to a negligible value after a sufficifntiy long $t j$ !ne. by detailed numerical solution ${ }^{1}$ of Eqs. (15). The on-axis intensity clearly dropg to a negligible value before the end of the pulse, and. as a consequence, the on-axis fluence saturates as the pulse width Increases, as can be seen in Fig * 17. The detailed temporal evolution of the spatial shape of the beam is shown in Figs. 18 and 19. Figure 18 is a three-dimensional plot of the laser intensity as a function of time and radius. Figure 19 shows the radial intensity profiles for increasing values of time. The opening up of a hole in the back of the pulse is clear from both Figs, 18 and 19.

Calculations of the lype represented in Figs. 17-19 become impractical if one is treating a

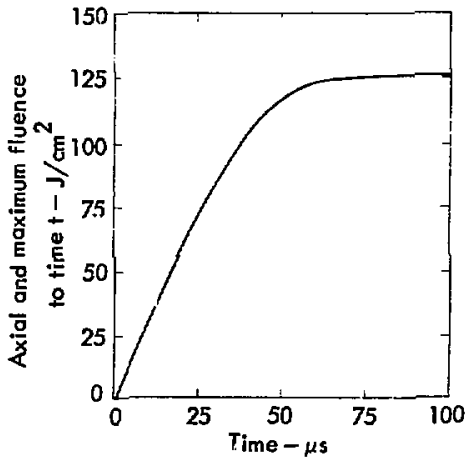

Fig. 37. Saturation of on-axis fluence due to strong pulse thermal blooming. 


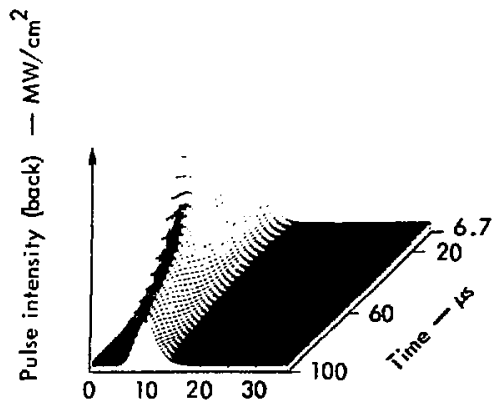

Radius - cm

Fig. 18. Three-dimensional plot of intensity as a function of time and radius corresponding to Figs. 16 and 17.

multipulse beam. The determination of nonisobarie contributions to the density is greatly simplified by the triangular pulse approximation, ${ }^{1}$ in which the dependence of the laser intensity on time is represented as an isosceles triangle with base equal to $2 \tau_{p}$. The density $1 s$ required only at time $t=\tau_{p}$, since
3

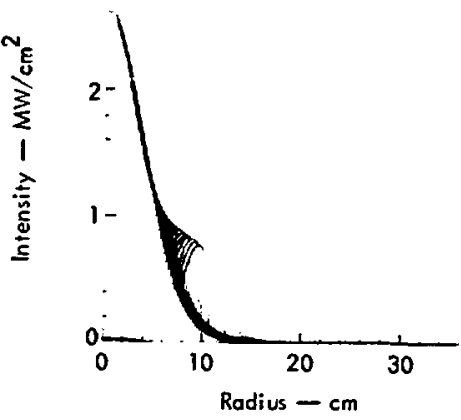

Fig. 19. Intensity as a function of radius $f \simeq i$ increasing time in pulse corresponding to Figs. 16 and 17.

the laser intensity is assumed to vanish for $t=0$ and $t \geq 2 \tau_{p}$.

The density change at $t=\tau_{p}$ can be evaluated analytically in terms of a finite Fourier series representtaction of the laser intensity. The Fourier transform of the nonisoba: $\because a l 1 y$ induced density change is $\tilde{p}_{1}^{s p}=-(\gamma-1) \frac{\alpha \tilde{T}_{p}}{2 c_{\varepsilon}^{2}}\left\{1-\frac{\sin ^{2}\left[\frac{1}{2} c_{s} \tau_{p}\left(k_{x}^{2}+k_{y}^{2}\right)^{1 / 2}\right]}{\left[\frac{1}{2} c_{s} \tau_{p}\left(k_{x}^{2}+k_{y}^{2}\right)^{1 / 2}\right]^{2}}\right\}$,

where $\tilde{I}$ is the spatial Fourier transform of the intensity. The corresponding density changes at the grid points are given by the discrete Fourier transform (DFT) expression

$$
p_{1}^{s p}(j \Delta x, k \Delta y)=(2 N)^{-2} \sum_{m, n=-N+1}^{N} \tilde{\rho}_{1}^{s p}\left(\frac{\pi m}{L}, \frac{\pi n}{L}\right) \exp \left(2 \pi i \frac{n i+n k}{2 N}\right) \text {, }
$$

$-33-$ 
where the basis functions are

pertodic on a square of side $2 L$.

Th1s allows for a buffer region that extends an additional distance $L$ in both the $x$ and $y$ directions fron the region of interest.

Comparison of the triangular nulse approxfmation and detalled pulse thermal-blooming calculations for Gaussian-shaped pulses in time have shown good agreement between the calculated fluences for weak or moderate thermal blooming.'

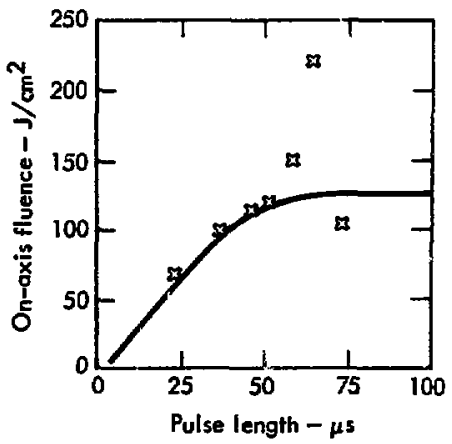

F1g. 20. On-axis fluence as a function of puise length, as calculated with triangular pulse approximation ( $\left.x^{\prime} s\right)$ and by detailed numerical solution of hydrodynamic equations for a square pulse in time (solld curve). The triangular pulse approximation breaks down as saturatedfluence condition sets in at $\tau_{p}=1.5 t$. Erratic behavior is fue to development of spikes in the Intensity pattern as a function of transverse position.

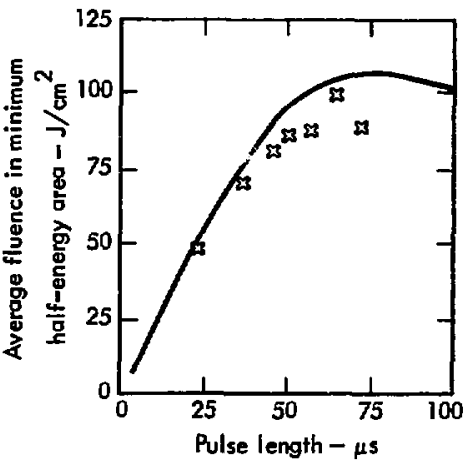

FIg. 21. Fluence averaged over minimum area containing one half of total beam energy, as a function of pulse length. Solfd curve is detalled calculation for square pulse, $x$ 's represent trlangular pulse approximation.

Figure 20 shows the on-axis fluence calculated for the previous example with the triangular pus.se approximation ( $x$ 's) and the detafled solution of Eqs. (15) for square pulses in time (solid line). Despite the difference in assumed pulse shapes, the agreement between the two types of calculation is very good up unt11 t1me $t \approx 50 \mu \mathrm{s}$, which Is well above the saturation time $t_{s}=38 \mu \mathrm{s}$ predicted by the perturbation theory of U1rich and Hayes ${ }^{8}$ based on the work of Aitken et al. ${ }^{9}$ Above $55 \mu \mathrm{s}$, or approximately $1.5 t_{B}$, the beam abruptly develops spikes In its transverse spat1al dependence; 
this clearly signals the breakdown of the trfangular pulse approximation, which must obviously fail when strong saturation behavior sets in.

Figure 21 shows the fluence averaged over the minfmum halfenergy area (the area within the one-half peak energy contour) calculated with the trlangular pulse approximation and with the detailed solution of Eqs. (15) for square pulses. Both calculations Increase Initially, reach a maximum, and then turn over with increasing time. This is in part due to the increase of the area within the one-half peak energy contour with time. There is, however, no point in believing the trlangular pulse approxidation beyond the time when the average fluence curve has reached a maximum, which also coincides with the onset of erratic behavior in the on-axis fluence (Fig, 20).

The perturbation theory ralluded to earlier 8,9 describes the on-axis fluence saturation for a br am that is Inttially Gaussian in shape and for a pulse shape that is square in time. In this theory, the expression for the on-axis intensity is

$$
I(t)= \begin{cases}I_{0}(z)\left(1-\frac{t^{3}}{t_{B}^{3}}\right) & t \leq t_{s} \\ 0 & t>t_{B},\end{cases}
$$

where $I_{0}(z)$ is the on-axis intensity for a Gausstan beam propagating in vacuum, or

$I_{0}(z)=\frac{I_{0}(0) e^{-\alpha z}}{D(z)}$.

Here $\alpha$ is the absorption coefflclent and

$$
D(z)=\left(1-\frac{z}{f}\right)^{2}+\left(\frac{a}{k a^{2}}\right)^{2},
$$

where $f$ is the focal distance and $a$ is the radius of the original Gaussian beam. The saturation time $t_{g}$ at on-axial position $z$ is given by

$t_{s}=\left[\frac{2 N(\gamma-1) \alpha z^{2} E p^{-\alpha z}}{3 \pi a^{6} D^{2}(z) \tau}\right]-1 / 3$

where $N$ is the refractivity, $E_{p}$ is the pulse energy, and $\tau_{p}$ is the pulse duration. Since the fluence cannot be increased for pulses longer than $t_{s}$, it can be argued that nothing is accomplished by making the pulse longer than $t_{s}$. The fluence must be maximized Instead by maximizing the product $I_{n}(z) t_{8}$ or, equivalently, by maximlzing $I_{0}(z)$. The maximum allowable value of $I_{0}(z)$ at point $z$ is normally determined by the condition that it not exceed the breakdown intensity, or 


$$
\max I_{0}(z)=I_{\mathrm{BD}} \text {. }
$$

This maximum allowable intenstey in turn determines a critical input pulse energy at $z=0$ given by

$E_{\text {crit }}=\pi a^{2} t_{s} I_{\mathrm{BD}} D(z) e^{\alpha z}$,

where Eq. (37) has been made use of, and where $t_{s}$ is calculated from

$t_{s}=\left[\frac{2 W(\gamma-1) \alpha z^{2} I_{\mathrm{BD}}}{3 \alpha^{4} D(z)}\right]^{-1 / 3}$.

If one is dealing with a multipulse laser with pulse-repetition frequency $v, E q$. (4I) can be used to define a critical input power with

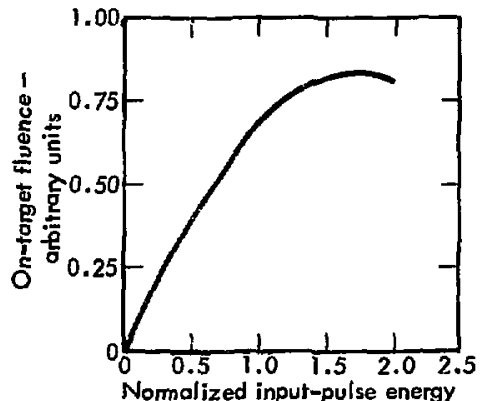

Fig. 22, On-target fluence from triangular pulse approximation averaged over area containing $(1-l / e)$ fraction oi total beam energy. Range $=1.5 \mathrm{~km}, \frac{1}{\mathrm{BD}}=1.6$ $\times 10^{6} \mathrm{~W} / \mathrm{cm}^{2}$.

$$
\begin{aligned}
P_{\text {crit }} & =\nu E_{\text {crit }} \\
& =\pi a^{2} v t_{s} I_{\mathrm{BD}} D(z) e^{\alpha z} .
\end{aligned}
$$

The self-consistency of the trlangular pulse approximation, on the other hand, prevents the on-axis intensity from ever becouing negative, but, as previously remarked, the triangular pulse approximation breaks down for pulse energies greater than the value that maximizes the space-averaged target fluence. For this pulse energy, the average and on-axis fluences shuuld be saturated, and further increases in pulse energy would give no return. Figures 22 and 23 have been calculated with the data on which

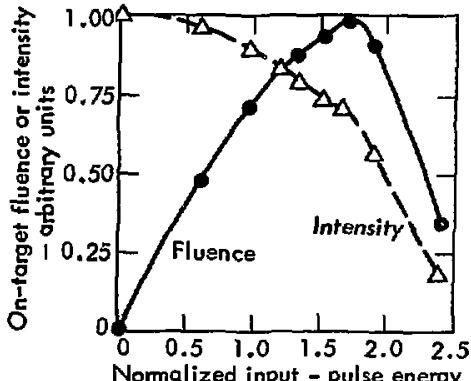

Fig, 23. On-target space-averaged fluence and intensity as functions of input pulse energy for triangular pulse approximation. Range $=2 \mathrm{~km}, I_{\mathrm{BD}}=3 \times 10^{6} \mathrm{~W} / \mathrm{cm}^{2}$. 
Figs, 16-21 are based, but with the following differences: the ranges for Figs, 22 and 23 are $1.5 \mathrm{~km}$ and $2.0 \mathrm{~km}$ respective1y; the values assigned (somewhat arbitrarily) to $I_{\mathrm{RD}}$ at these ranges are $3 \times 10^{6} \mathrm{~W} / \mathrm{cm}^{2}$ and $1.5 \times 10^{6} \mathrm{~W} / \mathrm{cm}^{2}$.

Both the on-target space-averaged fluence and intensity (Fig. 23) are plotted as functions of the input pulse energy normalized to $E_{\text {crit }}$ given in Eq. (42). The space averaging is over the area contained within the $1 / e$ energy contour. The indicated maxima of the average fluences in both Flgs. 22 and 23 occur at an Input pulse energy equal to $1.7 E$ crit $^{\text {. The space-averaged }}$ fluence curves in FIgs, 22 and 23 are smoother than those displayed in Fig. 20 because the former are averaged over larger areas. The scaling implications of the perturbation theory described in

Eqs. (36)-(42) are apparently valid for the triangular pulse approximation, although the maximum useful pulse energy predicted by the latter Is about $50 \%$ greater than that predicted by the perturbation theory.

In summary: the trlangular pulse approximation should provide reasonably accurate fluence results for pulse energies up to the values where strong thermal blooming saturates the on-axis fluence. The breakdown of the approximation will be indicated by the development of spikes in the transverse spatlal dependence of the beam intenstey as well as by a sharp falloff in the fluence averaged over some area as a function of pulse energy.

\section{Multipulse Thermal Blooming in the Triangular Pulse Approximation}

The propagation of a given pulse In a train is influenced by both the nontsobarte density changes discussed in the previous section and by the isobarlc density changes iue to heating by previous pulses in the train. But can the selfblooming and multipulse hlooming effects be treated independently?
If so, the results and discussion of the previous section suggest that, as tIme-averaged laser power is increased by lengthenfing the duration of the constituent pulses In the train, the time-averaged intensity on target should saturate at a value that is predictable from the saturation fluence for a single 
pulse, If $\langle I\rangle$ represents the timeaveraged intensity, the maximum ach Levable value of $\langle I\rangle$ for a given pulse-repetition rate should be expressible as

$$
\langle I\rangle_{\text {max }}=V F_{\text {sat }},
$$

where $F_{\text {sat }}$ is the single-pulse saturation fluence.

In order to test the hypothesis of the Independence of self and multipulse blooming, a set of calculations has been carried out with the following set of parameters: Start beam shape Gaussian, truncated at $1 / e^{2}$ radius

Range, $R$

$2.5 \mathrm{~km}$

Focal length/

$$
\text { range, } F / R \quad 1.0 \text { and } 1.2
$$

Wavelength, $\lambda \quad 10.6 \mu \mathrm{m}$

Absorption

$$
\text { coeffictent, } \alpha \quad 0.25 \mathrm{~km}^{-1}
$$

Aperture diamerer,

$2 a$ (Gaussian at

$$
\left.1 / e^{2}\right) \quad 21.2 \mathrm{~cm}
$$

Wind velocity, $v_{0} 10 \mathrm{~m} / \mathrm{s}$

Pulse-repetition

$$
\text { rate, } v \quad 33-1 / 3 \text { and } 50 \mathrm{~g}^{-1}
$$

raximum pulse

intensity at

receiver, $I_{\max } \quad 4.9 \mathrm{MW} / \mathrm{cm}^{2}$

Overlap number,

$$
N_{0}=20 v / v_{0} \quad 1.0,1.5
$$

Figure 24 shows the spaceaveraged single-pulse intensity $\bar{I}$ for $\nu=33-1 / 3 \mathrm{~s}^{-1}$ and $\nu_{0}=1$, with
$F / R=1.0$ and 1.2 , calculated as a function of input time-averaged power $\langle P\rangle=V E_{F^{*}}$. The curves have been calculated with and without the effects of pulse self-blooming. The curve without self-blooming for $F / R=1.2$ rises slightly with input power because of a very slight amount of pulse overlap. It is clear from Fig. 24, in any case, that thermal blooming is due almost entirely to self-blooming effects. The corresponding curves for space- and cimeaveraged target intensities $\langle\bar{T}\rangle$ are displayed in Fig. 25, where

$$
\langle\bar{I}\rangle=\bar{I} \tau \mathrm{T} .
$$

It is seen that $\langle\bar{T}\rangle$ with self-blooming rises initially, reaches a peak, and then falls. From the analysis of the previous section, we interpret the pcak values of $\langle\bar{I}\rangle$ as the saturated values.

Figure 26 shows $\bar{I}$ as a function of $\langle P\rangle$ for $\nu=50 \mathrm{~s}^{-1}$ and $v_{0}=1.5$, with $F / R=1.0$ and 1.2. Above $\langle P\rangle=$ $=0.5 \mathrm{MW}$, and an ewhancewent effect sets in that is greater in the case of the defocused beam. The corresponding curves for space- and timeaveraged target intensities are shown In F1g, 27.

A comparison of Figs. 25 and 27 is sumarized in Table 8 . It is seen that at $\nu=50 \mathrm{~s}^{-1}$ the power $\langle P\rangle_{\text {sat }}$ at which saturation of $\langle\bar{I}\rangle$ occurs is 


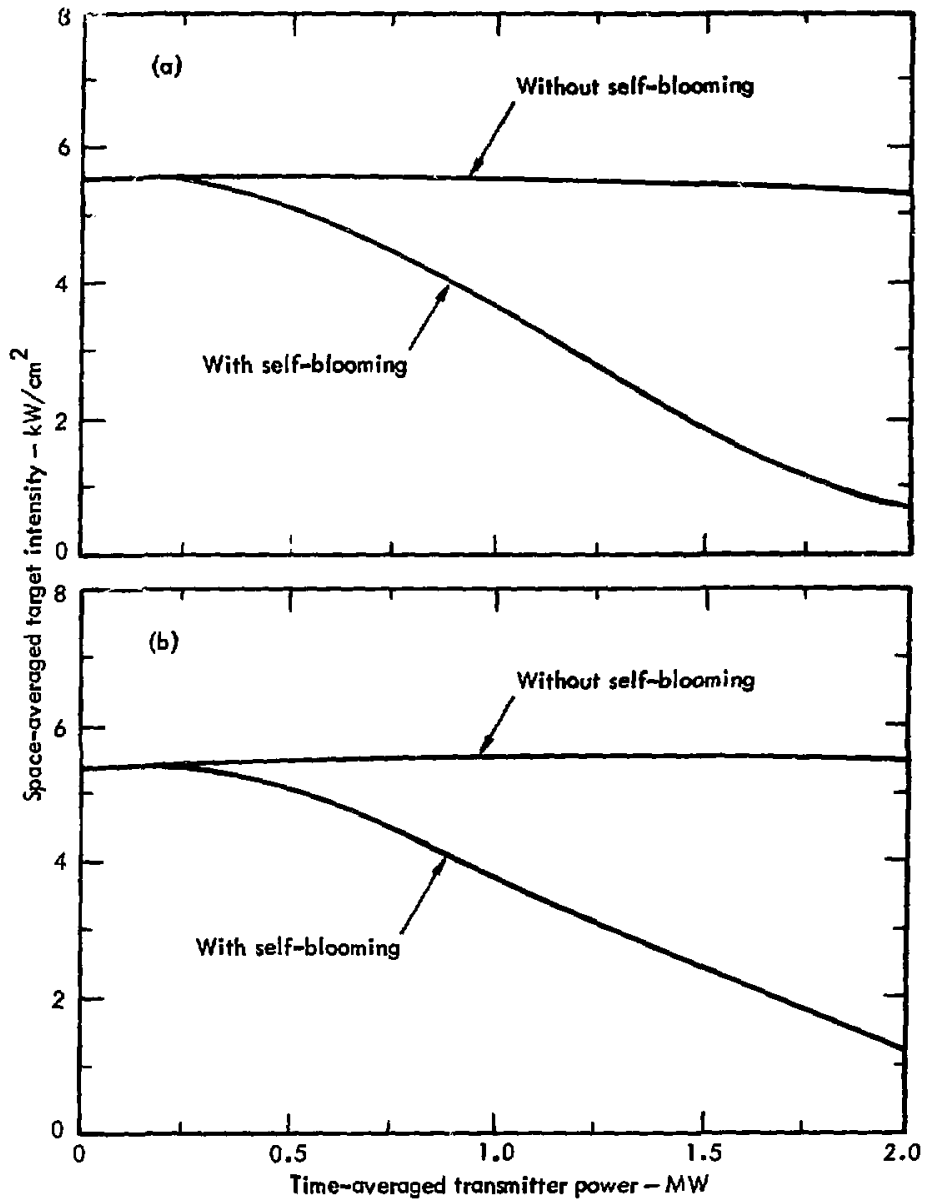

Fig. 24. Space-averaged Intensity on target as a function of time-averaged power at transmitter: $v=33-1 / 3 \mathrm{~s}^{-1}, N_{0}=1$. (a) $F / R=1.0$. (b) $F / R=1.2$. 


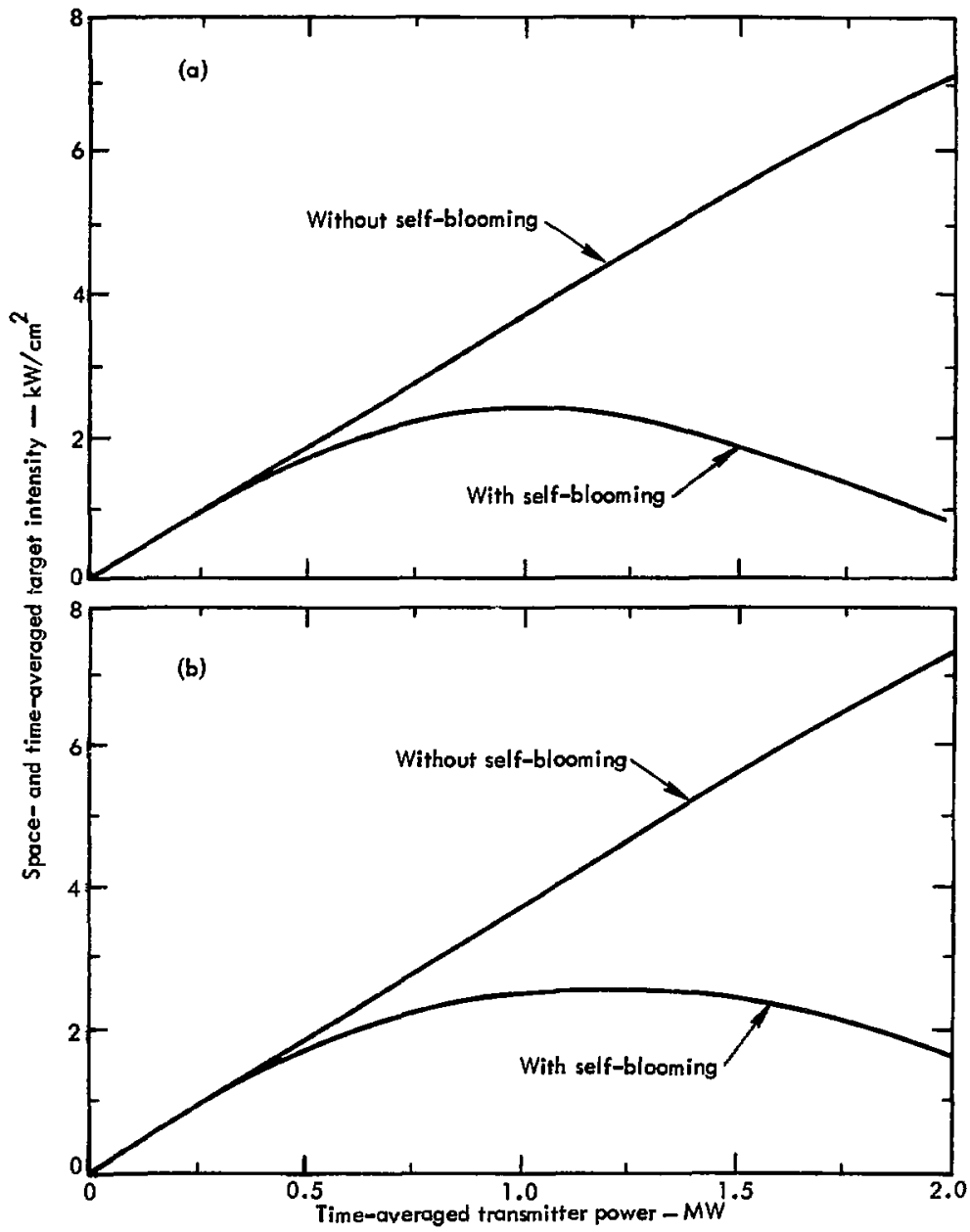

Fig. 25. Space- and time-averaged intensity on target as a function of timeaveraged power at transmitter: $v=33-1 / 3 \mathrm{~s}=1, N_{0}=1$. (a) $F / R=1.0$. (b) $F / R=1.2$. 


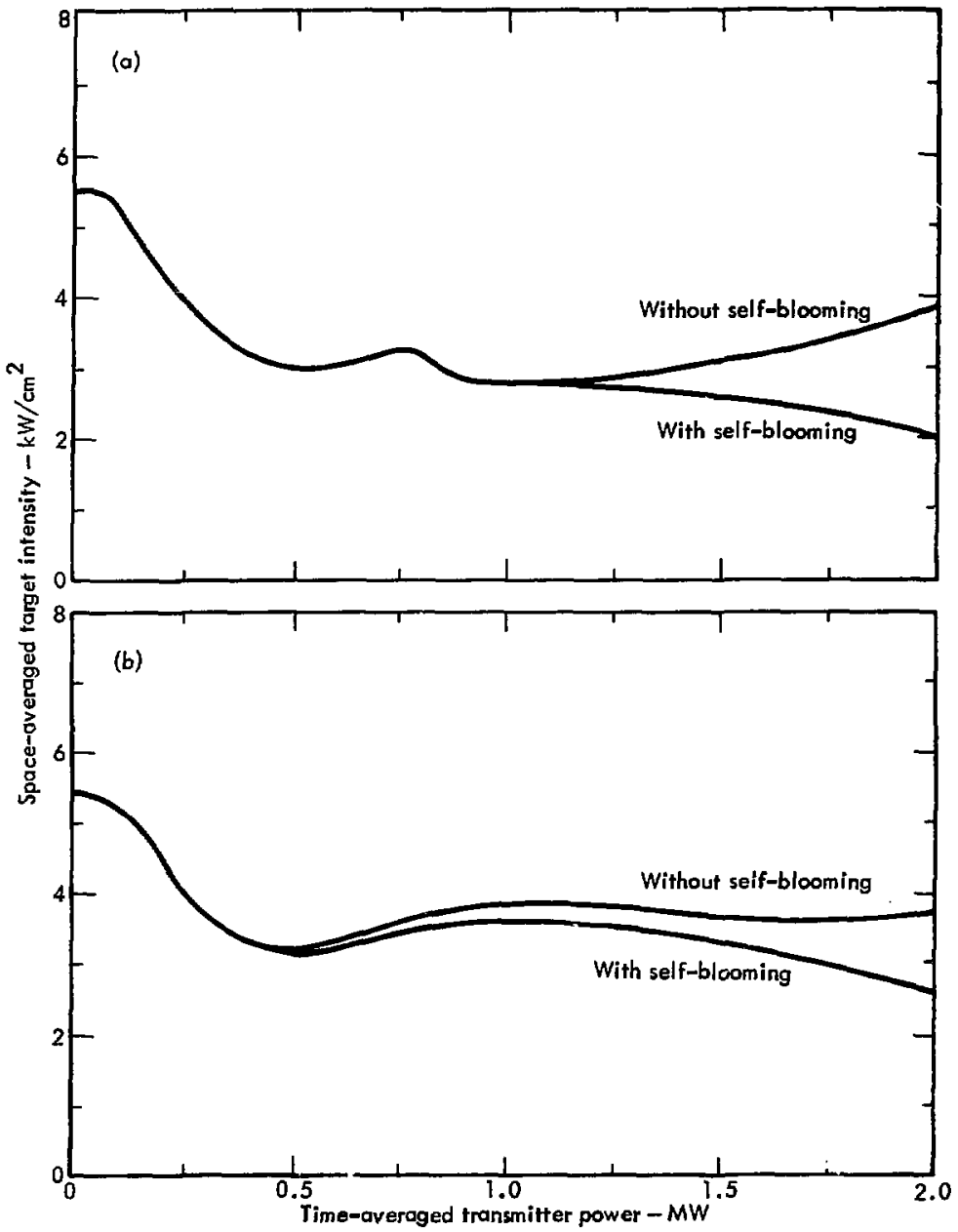

Fig. 26. Space-averaged intensity on target as a function of time-averaged power at transmitter: $v=50 \mathrm{~s}^{-1}, N_{0}=1.5$. (a) $F / R=1.0$. (b) $E / R=1.2$. 


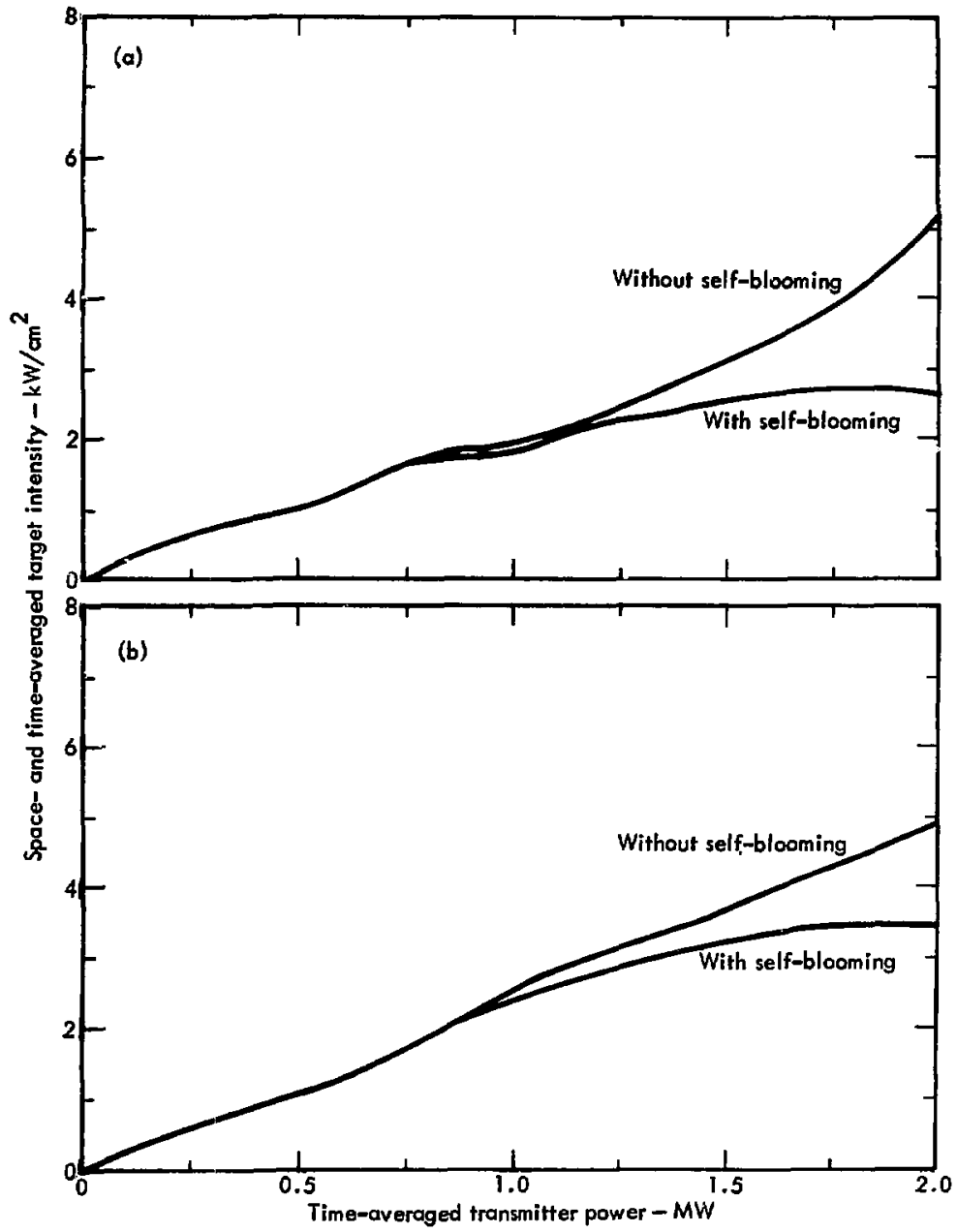

F1g. 27. Space- and timemaveraged 1ntenglty on target as a function of timeaveraged power at transmitter: $v=50 \mathrm{~s}^{-1}, N_{0}=1.5$. (a) $F / R=1.0$. (b) $F / R=1.2$. 
Table 8. Saturation of time- and space-averaged tarbet Intensity due to selfblooming.

\begin{tabular}{llll}
$\begin{array}{c}V \\
\left(\mathrm{~s}^{-1}\right)\end{array}$ & F/R & $\begin{array}{c}\langle P\rangle_{\text {sat }} \\
(\mathrm{MW})\end{array}$ & $\begin{array}{c}\langle\bar{T}\rangle_{\text {sat }} \\
\left(\mathrm{kW} / \mathrm{cm}^{2}\right)\end{array}$ \\
\hline $331 / 3$ & 1.0 & 1.0 & 2.4 \\
$331 / 3$ & 1.2 & 1.2 & 2.5 \\
50 & 1.0 & 1.75 & 2.7 \\
50 & 1.2 & 2.0 & 3.5 \\
\hline
\end{tabular}

higher for both values of $F / R$ than it is at $v=33-1 / 3 \mathrm{~s}^{-1}$. The corresponding saturation intensity values $\langle I\rangle$, sat are also greater at $v=50 \mathrm{~s}^{-1^{5 a t}}$ than at $v=33-1 / 3 \mathrm{~s}^{-1}$. If effects of self-blooming are not included, on the other hand, values of $\langle\bar{I}\rangle$ are always greater at a given value of $\langle P\rangle$ in the case of $v=33-1 / 3 \mathrm{~s}^{-1}$.

Unfortunately, we have no gulde to the accuracy of the triangular pulse approximation in the overlap case as we do in the nonoverlap case. But :he above results strongly suggest that the contributions of isobaric and nonisobarlc density changes to thermal blooming of multipulse beams are Interrelated, and that tine-averaged saturation incersities based on single saturation fluences may not be applicable for overlap numbers somewhat above 1 . In fact, overlapping isobaric density patterns may in certain situations actually override the effects of single-pulse nonisobaric density changes.

\section{Acknowledgment}

The authors are Indebted to C. H. Woods for the calculations of the effects of laser elevation on cw laser performance in Section 9 and for the effects of pulse thermal blooming on multipulse propagation in Section 11. 


\section{References}

1. J. A. Fleck, Jr., J. R. Morr Is, and M. Fe1t, Time-Dependent Propagation of High Energy Laser Beams Through the Atmosphere, Lawrence Livermore Laboratory, Rept. UCRL-51826 (1975); UCRL-77719 (1976) to ba published In Applied Physics.

2. R. T. Brown, P. J. Berger, F, G, Gebhardt, and H. C. Snith, Influence of Dead Zones and Transonic Slewing on Thermat Blooming, Untted Alrcraft Research Laboratory, East Hartford, Conn., Rept. N921724-7 (1974).

3. P. J. Berger, F. G. Gebhardt, and D. C. Smith, Themal Diooming Due to a Stagnation Zone in a Slewed Beam, United Alrcraft Research Laboratory, East Hartford, Conn., Rept. N921724-12 (1974).

4. P. J. Berger, P. B. Ulrich, J. T. Ulrich, and F. G. Gebhardt, "Transient Thermal Blooming of a Slewed Laser Beam Contalning a Regime of Stagnant Absorber," submitted to Applied Optics.

5. The possibility of such curved flow trajectorles in the neighborhood of the stagnation point was pointed out to one of the autinors by $B$. Hogge, private communication.

6. J, Wallace and J. R. Lilly, "Thermal Blooming of Repetitively Pulsed Laser Beams," J. Opt. Soc. Am. 64, 1651 (1974).

7. P. B. Ulrich and J. Wallace, $J$, Opt, Soc, Am. 63, 8 (1973).

B. P. B. Ulrich and J. N. Hayes, U.S. Naval Research Laboratory, Washington, D.C., unpublished Internal report (1974).

9. A. H. Altken, J. N. Hayes, and P. B. Ulrlch, "Thermal Blooming of Pulsed Focused Gaussian Laser Beans," Appl. Opt. 12, 193 (1973). 


\section{Appendix A: Adaptive Lens Transformation}

One key to the successful implementation of a laser-propagation code Is finding a coordinate transformation that keeps the laser beam away from the calculational mesh boundary and at the same time prevents the beam from contracting to an unreasonably small fraction of the total mesh area at the focus. If one is sciving the Fresnel equation by the finite Fourier transform method, one may alternatively view the problem in terms of cowplementarity: one wishes to find a transformation that simultaneously keeps the beam intensity small on the mesh boundaries in configuration space and keeps the Fourier spectrum small on the mesh boundaries in $k$-space. If these two conditions are met, one knows from sampling theory that the numerical solution lis highly accurate.

The Four-D code uses an automated procedure that is designed to keep the intensity centroid at the center of the mesh and the intensity-weighted r.m.s. values of $x$ and $y$ constant with propagation distance $z$. These conditiuss can be written

$$
\begin{aligned}
& \frac{\partial}{\partial z}\left\langle x_{i}\right\rangle_{I}=0, \\
& \frac{\partial}{\partial z}\left\langle\left(x_{i}-\left\langle x_{i}\right\rangle\right)^{2}\right\rangle_{I}=0, \\
& x_{1}=x, x_{2}=y,
\end{aligned}
$$

where

$$
\langle u\rangle_{I}=\frac{\int d x d y I(x, y) u}{\int d x d y I(x, y)} .
$$

Hereafter, all averayes will be assumed to be intensity-wetghted, and the subscript $I$ will be dropped.

Conditions (AI) also apply to the adaptive coordinate transformation of Bradley and Hermann, ${ }^{A l}$ which differs from the one employed in the Four $-D$

A1. L. C. Bradley and J. Hermann, "Change of Reference Navefront," Massachusetts Institute of Technology, Lincoln Laboratory, Lexington, Mass, unpublished internal report. 
code only in that it is preceded by a transformation to the coordinates of an arbitrary Gaussian beam propagating in vacuum. It should be evident, in any case, that such adaptive transformations are restricted to steady-state problems, since for time-dependent problems no single transformation will apply to a:l time values. To solve time-dependent problems one must employ a Talanov transformation that is optimized to all time values. This optimization is accomplished by $\therefore$ combination of trial and error and intuition.

The splitting algorlthm employed in the Four $-\mathrm{D}$ code can be written formally as

$$
\begin{aligned}
\varepsilon^{n+1} & =\exp \left(-\frac{i \Delta z}{4 k} \nabla_{1}^{2}\right) \exp \left(-\frac{i \Delta z}{2 k} \bar{x}\right) \exp \left(-\frac{i \Delta z}{4 k} \nabla_{1}^{2}\right) \varepsilon_{n}, \\
x & =k^{2}\left(n^{2}-1\right),
\end{aligned}
$$

where the mfddle exponential on the right-hand slde of Eq. (A2) contains the changes in phase resulting from hydrodynamic changes in citnsity, turbulence, etc. Immediately after this step in the calculation, a quadratic reference phase front is determined and is removed from $f$ by means of a Talanov transformation and a deflection of the beam coordinates. These operations are ca: ried out as part of the vacuun propagation step. During vacuun propagation the solution is advanced by solving

$$
2 i k \frac{\partial \mathscr{E}}{\partial z}=\nabla_{\perp}^{2} \mathscr{E}
$$

Equation (Al) can be written

$$
\begin{aligned}
& \left\langle x_{i}\right\rangle=\frac{1}{P} \int \mathrm{d} x_{1} \mathrm{~d} x_{2} x_{i}\left|\mathscr{E}\left(x_{1}, x_{2}, z\right)\right|^{2}, \\
& \left\langle x_{i}^{2},=\frac{1}{P} \int \mathrm{d} x_{1} \mathrm{~d} x_{2} x_{1}^{2}\left|\varepsilon\left(x_{1}, x_{2}, z\right)\right|^{2}, \quad i=1,2\right.
\end{aligned}
$$

where $P$ is the beam power given by

$$
P=\int \mathrm{di}_{1} \mathrm{~d} x_{2}\left|\mathscr{E}\left(x_{1}, x_{2}\right)\right|^{2} .
$$


By differenciating Eqs. (A4a) and (A4b) with respect to 2 and making use of the Fresuel equation (A3), one obtains the following relations:

$$
\begin{aligned}
& \frac{\partial}{\partial z}\left\langle x_{i}^{2}\right\rangle=-\frac{2}{k^{P}} \int d x_{1} d x_{2}\left|\mathscr{E}\left(x_{1}, x_{2}, z\right)\right|^{2} x_{i} \frac{\partial}{\partial x_{i}} \phi\left(x_{1}, x_{2}, z\right), \\
& \frac{\partial}{\partial z}\left\langle x_{i}\right\rangle=-\frac{1}{k P} \int d x_{1} d x_{2}\left|\mathscr{E}\left(x_{1}, x_{2}, z\right)\right|^{2} \frac{\partial}{\partial x_{i}} \phi\left(x_{1}, x_{2}, z\right),
\end{aligned}
$$

wherc the phase $\phi\left(x_{1}, x_{2}, z\right)$ is defined by

$$
\phi\left(x_{1}, x_{2}, z\right)=\operatorname{In}\left[\ln \mathscr{E}\left(x_{1}, x_{2}, z\right)\right] .
$$

In $k$-space one can similarly derive

$$
\begin{gathered}
\frac{\partial}{\partial z}\left\langle x_{i}\right\rangle=\frac{1}{k P} \iint d \kappa_{1} d \kappa_{2} \kappa_{i}\left|\tilde{E}\left(\kappa_{1}, \kappa_{2}, z\right)\right|^{2}=\frac{\left\langle\kappa_{i}\right\rangle}{k}, \\
\frac{\partial}{\partial z}\left\langle x_{i}{ }^{2}\right\rangle=-\frac{2}{k P} \operatorname{In} \iint d \kappa_{1} d \kappa_{2} \kappa_{i}\left|\widetilde{E}\left(\kappa_{1}, \kappa_{2}, z\right)\right|^{2} \\
\times \frac{\partial}{\partial \kappa_{i}} \psi\left(\kappa_{1}, \kappa_{2}, z\right),
\end{gathered}
$$

where $\tilde{E}\left(\kappa_{1}, \kappa_{2}, z\right)$ is the Fourier transform of $\mathscr{E}\left(x_{1}, x_{1}, z\right)$, and

$$
\psi\left(\kappa_{1}, \kappa_{2}, \approx\right)=\operatorname{Im}\left[\operatorname{In} \mathscr{E}\left(\kappa_{1}, \kappa_{2}, z\right)\right]
$$

We now wish to determine a phase front that will preserve the following conditions:

$$
\begin{aligned}
& \frac{\partial}{\partial z}\left\langle x_{i}\right\rangle=\dot{0}, \\
& \frac{\partial}{\partial z}\left\langle\left(x-\left\langle x_{i}\right\rangle\right)^{2}\right\rangle=0,
\end{aligned}
$$


Equation (A9b) is equivalent to

$$
\left.\frac{\partial}{\partial z}\left\langle i_{i}{ }^{2}\right\rangle-2<x_{i}\right\rangle \frac{\partial}{\partial z}\left\langle x_{i}\right\rangle=0 .
$$

From Eqg, (A9) and (A6) one obtalns

$$
\begin{aligned}
& \frac{\partial}{\partial z}\left\langle x_{i}\right\rangle=-\frac{i}{k}\left\langle\frac{\partial}{\partial x_{i}} \phi\right\rangle=0, \\
& \frac{\partial}{\partial z}\left\langle x_{i}^{2}\right\rangle=-\frac{i}{k}\left(x_{i} \frac{\partial}{\partial x_{i}} \phi\right\rangle=0 .
\end{aligned}
$$

Thus the reference phase front must satisfy

$$
\begin{aligned}
& \left\langle\frac{\partial}{\partial x_{i}} \phi\right\rangle=0, \\
& \left\langle\left(x_{i}-\left\langle x_{i}\right\rangle\right) \frac{\partial}{\partial x_{i}} \phi\right\rangle=0 .
\end{aligned}
$$

Let us define a new phase varlable:

$$
\begin{aligned}
\phi^{\prime}\left(x_{1}, x_{2}, z_{n+x_{2}}\right)=\phi_{0}\left(x_{1}, x_{2}, z_{n+x_{2}}\right) \\
\\
+\sum_{i=1}^{2}\left[\alpha_{i}\left(x_{i}-\left\langle x_{i}\right\rangle\right)^{2}+\beta_{i}\left(x_{i}-\left\langle x_{i}\right\rangle\right)\right],
\end{aligned}
$$

where $\phi_{0}\left(x_{1}, x_{2}, z_{n+\frac{1}{2}}\right)$ represents the phase $\phi\left(x_{1}, x_{2}, z_{n+\frac{1}{2}}\right)$ at $z_{n+\frac{1}{2}}$ before the vacuum propagation operator has been applied, and where $\alpha_{i}$ and $\beta_{i}$ are determined so as to make conditions (A11a) and (A11b) hold for the phase front $\phi^{\prime}\left(x_{1}, x_{2}, z_{n+1}\right)$. From Eq. (Al1a) we obtaln

$\left\langle\frac{\partial}{\partial x_{i}} \phi^{\prime}\left(x_{1}, x_{2}, z_{n+\frac{1}{2}}\right)\right\rangle=2 \alpha_{i}\left\langle x_{i}-\left\langle x_{i}\right\rangle\right\rangle+\beta_{i}$

$$
+\left\langle\frac{\partial}{\partial x_{i}} \phi_{0}\left(x_{1}, x_{2}, z_{n+\frac{1}{2}}\right)\right\rangle
$$


or

$$
B_{i}=-\left\langle\frac{\partial}{\partial x_{i}} \phi_{0}\right\rangle=\frac{\left\langle k_{x}\right\rangle}{k}
$$

From (Allb) we obtain

$$
\begin{aligned}
& \left\langle\left(x_{i}-\left\langle x_{i}\right\rangle\right) \frac{\partial}{\partial x_{i}} \phi^{\prime}\left(x_{1}, x_{2}, z_{n+\frac{1}{2}}\right)\right\rangle=2 \alpha_{i}\left\langle\left(x_{i}-\left\langle x_{i}\right\rangle\right)^{2}\right) \\
& \left.+\beta_{i}<x_{i}-\left\langle x_{i}\right\rangle\right\rangle=0 \\
& \alpha_{i}=\frac{\left(\left(x_{i}-<x_{i}>\right) \frac{\partial \phi_{0}}{\partial x_{i}}\right)}{2\left\langle\left(x_{i}-\left\langle x_{i}\right\rangle\right)^{2}\right\rangle}, \quad i=1,2 .
\end{aligned}
$$

Equatlons (Al3) and (A14), which determine the desired reference phase-front parameters ( $A 12$ ), can be shown to be completely equivalent to the relations used by Bradley and Hetmans. $A 1$

If the optimal phase front $\phi^{\prime}$ is now substituted for the original phase front $\phi_{0}$ at $z_{n+\frac{1}{2}}$, the phase increment

$$
\phi_{0}-\phi^{\prime}=-\sum_{i=1}^{2}\left[\alpha_{i}\left(x_{i}-\left\langle x_{i}\right\rangle\right)^{2}+\beta_{i}\left(x_{i}-\left\langle x_{i}\right\rangle\right)\right]
$$

must be compensated for In some way in vider to preserve the original field. The quadratic contribution in (A15) is compensated for by a generalized Talanov transformation, which involves a rescaling of $\mathscr{E}$, the mesh, and $\Delta z$, according to

$$
\begin{aligned}
\mathscr{E}(x, y, z) & =\frac{1}{\left(1-\frac{\Delta z}{z_{x}}\right)^{\frac{1}{2}}\left(1-\frac{\Delta z}{z_{x}}\right)^{\frac{1}{2}}} \\
& \times \mathscr{E}\left(\frac{x}{1-\frac{\Delta z}{z_{x}}}, \frac{z}{1-\frac{\Delta z}{z_{y}}}, z\right) \exp \left[i \frac{k}{2}\left(\frac{x^{2}}{z_{x}-\Delta z}+\frac{y^{2}}{z_{y}-\Delta z}\right)\right],
\end{aligned}
$$


where

$$
\begin{aligned}
& \tilde{E}\left(\kappa_{x}, \kappa_{y}, z\right)=\widetilde{\mathscr{E}}\left(\kappa_{x}, \kappa_{y}, z-\Delta z\right) \exp \left[i\left(\frac{z_{1} \kappa_{x}^{2}}{2 k}+\frac{z_{2} \kappa_{y}^{2}}{2 k}\right)\right], \\
& z_{1}=\frac{z}{1-\left(\Delta z / z_{x}\right)}, \\
& z_{2}=\frac{z}{1-(\Delta z / z y)} .
\end{aligned}
$$

The generalized focal lengths $z_{x}$ and $z_{y}$ are determined by combining the reciprocals of the current focal lengths,

$$
\begin{aligned}
& z_{x}^{c}=2 \alpha_{1} / k, \\
& z_{y}^{c}=2 \alpha_{2} / k,
\end{aligned}
$$

with those remaining from previous propagation steps (see argument of exponential in Eq. (Al6a)).

The linear term in (A15) corresponds to solving Eq. (A3) in a coordinate system that has been rotated in $x-y-z$ space. If this rotation is assumed to be small, it can be represented by a net deflection in the $x$ and $y$ coordinates given by

$$
\begin{aligned}
& \delta x=-\left(\beta_{1} / k\right) z, \\
& \delta y=-\left(\beta_{2} / k\right) z .
\end{aligned}
$$

The contribution $\Sigma_{i} \beta_{i}\left(x_{i}-\left\langle x_{i}\right\rangle\right)$ must a lso be added to $\phi_{0}$ before the vacuut propagation calculation, but this operation may correspond to a translation of the Fourler transform $\mathscr{E}\left(\kappa_{x}, \kappa_{y}\right)$ by a nonintegral number of steps on the 
$k$-space mesh. In order to avoid this, $\beta_{1} / \Delta \kappa_{x}$ and $\beta_{2} / \Delta \kappa_{y}$ are both rounded off to the nearest integer, and $\mathscr{E}\left(K_{x}, K_{y}\right)$ is then translated on its mesh in the $x$ and $y$ directions by the corresponding number of steps.

The numerical implementation of Eqs. (A13) and (A14) requires the following computations, where $j$ and $k$ represent the numerical coordinates of the mesh points:

$$
\begin{aligned}
& E=\sum_{j, k}\left|E_{j k}\right|^{2} \\
& <x>=\frac{1}{E} \sum_{j, k} x_{j}\left|\circ_{j k}\right|^{2}, \\
& \left\langle(x-\langle x\rangle)^{2}\right\rangle=\frac{1}{E} \sum_{j, k} x_{j}^{2}\left|\mathscr{E}_{j k}\right|^{2}-x^{2}, \\
& \sigma_{x}=\frac{\sum_{j, k} \kappa_{j}\left|\tilde{\delta}_{j k}\right|^{2}}{\sum_{j, k}\left|\tilde{E}_{j k}\right|^{2}},
\end{aligned}
$$

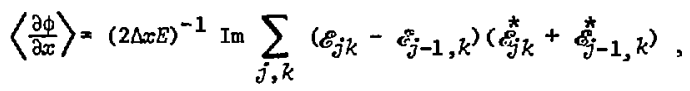

$$
\begin{aligned}
& \left\langle(x-\langle x\rangle) \frac{\partial \phi}{\partial x}\right\rangle=(2 \Delta x E)^{-1} \\
& \times \operatorname{Im} \sum_{j, k} x_{j}\left(\mathscr{E}_{j k}-\mathscr{E}_{j-1, k}\right)\left(\mathscr{E}_{j k}^{*}+\dot{\mathscr{E}}_{j-1, k}^{*}\right)-\langle x\rangle\left\langle\frac{\partial \phi}{\partial x}\right\rangle .
\end{aligned}
$$


The computations involving the variable $y$ are carried out in an analogous manner. In the calculation of the average phase derivative, the phase derivative is monitored at each point and limitcd in magnitude to a fraction of $\pi$. This prevents rapid phase fluctuations near the mesh boundary, where intensitles may be weak, from contributing disproportionately to the average. 


\section{Appendix B: An Adaptive Algorithm for Selecting the Axial Space Increment}

It Is desirable to have the code select the next axlal space increment $\Delta z$ at a given axial position on the basis of requirements for numerlcal accuracy In the solution of the wave equation. The numerical acciracy of the vacuum propagators in the symmetrically split solution operator,

$$
\mathscr{E}^{n+1}=\exp \left(-\frac{i \Delta z}{4 k} \nabla_{\perp}^{2}\right) \exp \left(-\frac{i \Delta z}{2 k} \vec{x}\right) \exp \left(-\frac{i \Delta z}{4 k} \nabla_{\perp}^{2}\right) \mathscr{E}^{n},(B 1)
$$

Is independent of $\Delta z$ if the solution is based on a discrete Fourler transform. The imposition of the phase front,

$$
\Delta \phi=-\frac{\Delta z \bar{X}}{2 \bar{k}}
$$

at $z=z_{n+\frac{1}{2}}$, which is equivalent to passing tie beam through a Iens, will make the solution meaningless if any of the transverse zone-to-zone phase differences violate

$$
\begin{aligned}
& \left|\delta_{x}(\Delta \phi)\right| \leqslant f \pi, \\
& \left|\delta_{y}(\Delta \phi)\right| \leqslant f \pi, \\
& 0<f \leq 1 .
\end{aligned}
$$

It w111 always be necessary then to restrict the value of $\Delta a$ so that conditlons (B3) are met. While violating conditions (B3) destroys the numerical integrity of the solution, satisfying them does not completely guarantee accuracy, since errors can also result from the noncommutation of $\nabla_{\perp}{ }^{2}$ and $\bar{X}$, and from upgrading $\bar{x}$ too infrequently. These errors must be controlled externally by inputting a maximum allowable value of $\Delta z$.

In practlce, part of the effect of the phase front (B2) is removed by the adaptive lens transformation. It would therefore be too restrictive to $11 m 1 t \Delta z$ on the basls of conditions (B3). As an alternative one can restrict the value of $\Delta \mathrm{s}$ sa as to control transverse gradients in the phase varlable 


$$
\theta=\sum_{i=1}^{2} \alpha_{i}\left(x_{i}-\left\langle x_{i}\right\rangle\right)^{2}-\frac{k}{2} \frac{\partial \varepsilon}{\partial \rho} \Delta z^{n} \rho_{1},
$$

which is that part of the nonlinear phase front at $z_{n+\frac{1}{2}}$ that cannot be removed by the adaptive lens transformation. The next spacial increment $\Delta z^{n+1}$ is then chosen in terms of the current value $\Delta z^{n}$ by means of the relation

$$
\Delta z^{n+1}=\frac{f \Delta z_{\pi}^{n}}{\max || \theta_{j, k+1}-\theta_{j k}|,| \theta_{j+1, k}-\theta_{j k}||_{I_{j k}>f^{\prime} I_{\max }}} .
$$

The arguments of the naximum function in the denominator of expression (B5) are restricted to those mesh points where the intensity is greater than a certain fraction $f^{\prime}$ of the maximum Intensity. The final value of $\Delta z^{n+l}$, however, must satisfy the additional constraints:

$$
\begin{aligned}
0.8 \Delta z^{n} & \leq \Delta z^{n+1} \leq 1.2 \Delta z^{n}, \\
\Delta z_{\min }<\Delta z^{n+1} \leq \Delta z_{\max }, & z_{T} \sqrt{2 f_{D}} \\
\Delta z^{n+1} & \leq \frac{z_{T}}{\sqrt{2 f_{D}}+\frac{z^{2}}{k\left[\left\langle(x-\langle x\rangle)^{2}\right\rangle+\left\langle(y-\langle y\rangle)^{2}\right\rangle\right]}},
\end{aligned}
$$

with (B6) taking precedence over (B5), (B7) over (B6), and (B8) over (B7). In Eq. (B8), $f_{D} \approx 0.005$ is an input fraction and $z_{T}=\min \left(\left|z_{x}\right|,\left|z_{y}\right|\right)$. Condition (B8) is designed to reduce $\Delta z$ near a focus, where the geometricoptics scallng of the mesh by the Talanov transformation may result in an excessive shrinkage of the mesh. By updating the Talanov transformation sufficlently often, one can usually avold a geometric-optics catastrophe.

The adaptive $z-s t e r$ algortthm Just described adds greatly to the conventence of running problems; 1 t often improves problem runing time, and avolds large nonlinear phase changes that can invalidate the calculation. It should not, however, be regarded as a panacea. For suffictently high beam power and strong enough thermal blooming, the criterla (B5)-(B8) can 
be satisfled and yet the problem st11l goes bad. In such cases, large nonquadratic trancverse zone-to-zone phase differences can accumulate over many $z$-steps. 


\section{Appendix C: Treatment of Multiline Absorption}

The treatment of multiline absorption in the Four-D code follows the method of Hogge. ${ }^{C 1}$ The basic assumptions are that all 1Ines operate with the same transverse mode structure in the laser and that the line frequencles are near enough to each other so that the fleld for each 1 ine will be affected in the same way by the atmospheric density distribution. Thus at each posttion $z$, for the ith line $\mathscr{E}_{i}(z) \propto \mathscr{E}(z)$, and the field will be comp.letely characterized by the fractions $f_{i}(z)$ of the total power $P(z)$ that are found in each line.

At $z=0$ one has

$$
P_{i}(0)=f_{i}(0) P(0)
$$

and at position $z$

$$
\begin{aligned}
P_{i}(z) & =P_{i}(0) e^{-\alpha_{i} z}, \\
P(z) & =\sum P_{i}(z)=P(0) \quad \sum f_{i}(0) e^{-\alpha_{i} z}, \\
f_{i}(z)= & \frac{f_{i}(0) e^{-\alpha_{i} z}}{\sum_{i} f_{i}(0) e^{-\alpha_{i} z^{*}}}
\end{aligned}
$$

Thus,

$$
I_{i}(a)=f_{i}(a) I(a)
$$

and the energy-deposition rate per unit $v$ lume is given by

C1. C. B. Hogge, "A Comparison of Several High Energy Laser Systems with Emphasis on Propagation Aspeets," in Laser Digest, AFWL-TR-75-140 (May 1975). 


$$
\sum_{i} \alpha_{i} I_{i}(z)=I(z) \sum_{i} \alpha_{i} f_{i}(z)
$$

in which

$$
\sum_{i} \alpha_{i} f_{i}(z)=\bar{\alpha}(z)
$$

can be interpreted as an average cross section throughout the calculation. Tables $C 1$ and $C 2$ show values for $\bar{\alpha}(z)$ as a function of $z$ for the DF Iine data found in Ref. C2. Clearly, for DF the effect of including all line absorption details leads to a very small correction even at $10 \mathrm{~km}$.

\begin{tabular}{|c|c|c|c|c|}
\hline Line ID & $\begin{array}{l}\text { Line frequency } \\
\qquad\left(\mathrm{cm}^{-1}\right)\end{array}$ & $\frac{\text { Fraction }}{z=0}$ & $\frac{\text { total pioner }}{z=1.0 \text { tom }}$ & $\begin{array}{l}\text { Absorption } \\
\text { coefficient } \\
\text { (km-1) }\end{array}$ \\
\hline $\begin{array}{l}4-3,7 \\
3-2,10 \\
4-3,6 \\
3-2,9 \\
4-3,5 \\
3-2,8 \\
3-2,7 \\
2-1,10 \\
3-2,6 \\
2-1,9 \\
3-2,5 \\
2-1,8 \\
2-1,7 \\
2-1,6 \\
1-0,9 \\
2-1,5 \\
1-0,8 \\
1-0,7 \\
1-0,6 \\
1-0,5\end{array}$ & $\begin{array}{c}- \\
2496.77 \\
- \\
2521.81 \\
- \\
2546.42 \\
2570.51 \\
2580.10 \\
2594.25 \\
2605.80 \\
2617.44 \\
2631.06 \\
2655.85 \\
2680.17 \\
2691.61 \\
2703.99 \\
2717.54 \\
2743.00 \\
2767.97 \\
2792.43\end{array}$ & $\begin{array}{r}0.01040 \\
.00590 \\
.02130 \\
.01330 \\
.01040 \\
.04750 \\
.06380 \\
.00910 \\
.08970 \\
.03180 \\
.05630 \\
.08450 \\
.09040 \\
.1 .3040 \\
.03230 \\
.04000 \\
.06440 \\
.08740 \\
.08370 \\
0.02740\end{array}$ & $\begin{array}{r}0.01006 \\
.00636 \\
.02060 \\
.01632 \\
.01006 \\
.05401 \\
.06108 \\
.00813 \\
.11944 \\
.03786 \\
.07960 \\
.10460 \\
.05918 \\
.12864 \\
.02557 \\
.05300 \\
.03629 \\
.08434 \\
.06178 \\
0.02310\end{array}$ & $\begin{array}{r}0.06000 \\
.04920 \\
.06000 \\
.03620 \\
.06000 \\
.04380 \\
.06100 \\
.06790 \\
.02800 \\
.03920 \\
.02200 \\
.03530 \\
.09900 \\
.05800 \\
.08000 \\
.02850 \\
.11400 \\
.06020 \\
.08700 \\
0.07370\end{array}$ \\
\hline
\end{tabular}

Table C1. Line-by-1ine absorption-coeffictent. data.

C2. R. K. Long, F. S. Mills, and G. L. Trusty, CaZaulated Absorption Coefficients for DF Laser Frequencies, Ohio State University ElectroScience Laboratory, Rept. RADC-TR-73-389. 
Table C2, Mean absorption coefficlent as a function of distance.

\begin{tabular}{ccc}
$\begin{array}{c}\text { Propagation } \\
\text { distance } \\
(\mathrm{km})\end{array}$ & $\begin{array}{c}\text { Mean } \\
\text { absorption } \\
\text { coefficient } \\
\left(\mathrm{km}^{-1}\right)\end{array}$ & $\begin{array}{c}\text { Remaining } \\
\text { power } \\
\text { fraction } \\
\text { in beam }\end{array}$ \\
\hline 0 & 0.06001 & 1.00000 \\
1 & 0.05931 & 0.94209 \\
2 & 0.05862 & 0.83815 \\
3 & 0.05793 & 0.83787 \\
4 & 0.05726 & 0.79097 \\
5 & 0.05660 & 0.74720 \\
7 & 0.05594 & 0.70632 \\
8 & 0.05530 & 0.66811 \\
9 & 0.05467 & 0.63236 \\
10 & 0.05404 & 0.59891 \\
\hline
\end{tabular}




\section{Appendix D: Characterization of Nondiffraction-Limited Beams}

In the absence of detailed a priori Informatton regarding the exact mode content of a beam, several models of nondiffraction-limited beam behavior can be applied with the Four $-D$ code.

The simplest of these, which requires no spectal coding, is wavelength scaling, wherein the laser wavelength is multiplied by a number equal to the beam quality factor. Wavelength scaling glves the correct vacuum peak intensity, although it may incorrectly represent the vacuum focal-spot size. It represents, in any case, a prescription whose accuracy needs to be evaluated ad hoc for each specific application. While it has been useful in a variety of applications, it does not properly account for discrepancfes between calculations and stagnation blooming experiments in vertical absorption cells. ${ }^{\mathrm{Dl}}$

Agreement between measured data and calculations for these experiments is Improved, on the other hand, by adding spherical aberration to the faitial beam in such a way that the vacum focal-spot size is correctly reproduced (see Fig. D1). The spherical aberration contribution to the Initial phase can be represented as

$$
\phi^{\mathrm{SA}}=\frac{2 \pi A}{\sigma_{x}^{2}}\left(x^{2}+y^{2}\right)^{2},
$$

where $A$ represents the number of waves of aberration at radius $\sigma_{x}$.

A third model of nondfffraction-limited vehavior is due to Hogge et $a z$. D2 This model is based on the assumption that the fnitial beam can be represented by

$$
\mathscr{E}(x, y, 0)=\sigma_{0}(x, y) e^{i \phi(x, y)},
$$

where the phase aberration $\phi(x, y)$ is a Gaussian random varlable, arising frow lager-medium inhomogeneitfes, mfrror imperfect.ons, etc. If it is assumed that the correlation function for phase fluctuations is

D1. J.A. Fleck, Jr., J. R. Morris, and M. D. Felt, Time-Dependent Propagation of High Energy Laser Beams Through the Atm :phere, Lawrence Livermore Laboratory, Rept. JCPL-51826 (1975).

D2. C, B. Hogge, R, R. Butts, and H. Burlakoff, App 2 . Opt. 13, 1065 (1974). 


$$
c_{\phi}(r)=\sigma_{\phi}^{2} \exp \left(-\frac{r^{2}}{2 z_{0}^{2}}\right)
$$

where $r^{2}=x^{2}+y^{2}, \sigma_{\phi}^{2}$ is the variance of $\phi$, and $\tau_{0}$ ts the phase coherence leugth, then the spectrum of the phase fluctuations is given by

$$
\tilde{c}_{\phi}\left(k_{\phi}\right)=\frac{\sigma_{\phi}^{2} z_{0}^{2}}{2 \pi} \text { exp }\left(-\frac{z_{0}^{2} k_{\phi}^{2}}{2}\right) .
$$

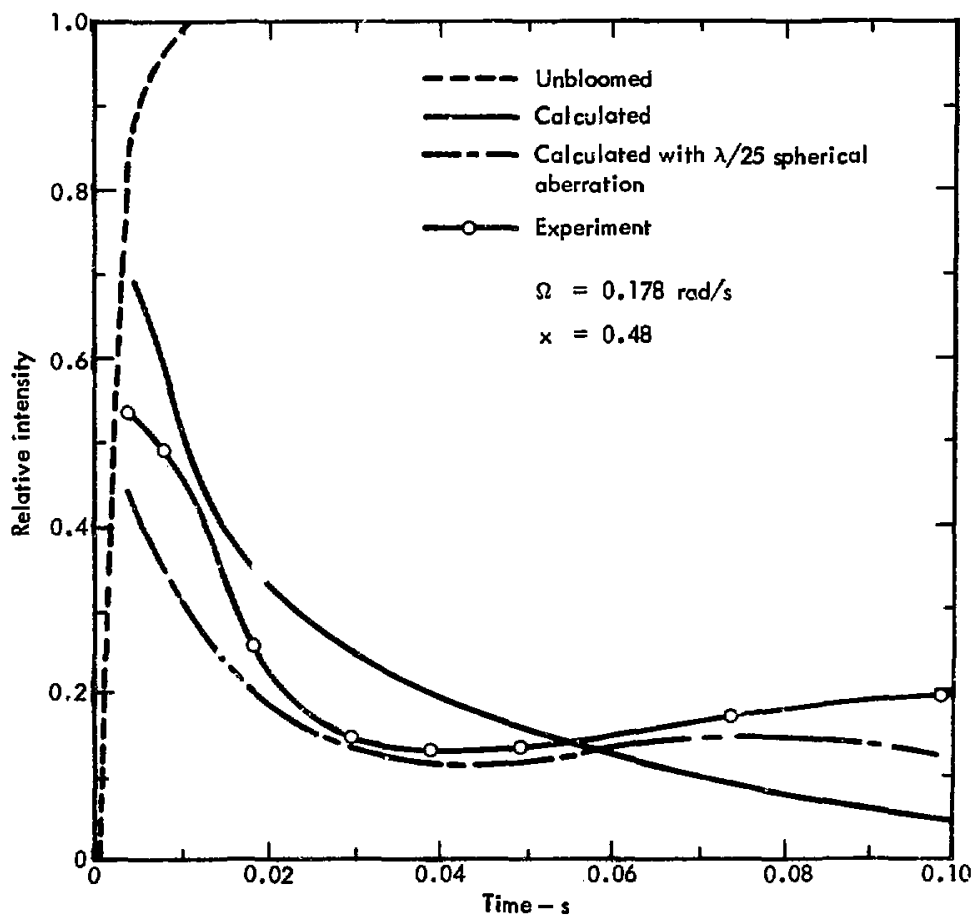

$\mathrm{Fi}_{\mathrm{b}}, \mathrm{D} 1$. Intensity on target after passing through stagnation zone. Comparison between experiment and calculation with and without spherical aberration. (Data from P. J. Berger, F. G. Gebhardt, and D. Smith, Thermal Blooming Due to a Stagnation Zone in a Slewed Beam, United Aircraft Research Laboratory, East Hartford, Conn., Rept. N921724-12 (1974).) 
Using the method of phase screens, one can obtain the Fourfer transform of the phase $\phi(x, y)$ to be used in Eq. (D1) in the following form:

$$
\phi\left(\underline{k}_{\phi}\right)=\left(\frac{a^{\prime}\left(\underline{k}_{\phi}\right)+i a^{\prime \prime}\left(\underline{k}_{\phi}\right)}{\sqrt{2}}\right) \widetilde{c}_{\phi}^{\prime-1}\left(\underline{k}_{\phi}\right),
$$

where $a^{\prime}$ and $a^{\prime \prime}$ art Gaussian random variables with variance 1, and where

$$
\begin{aligned}
& a^{\prime}\left(\underline{k}_{\phi}\right)=a^{\prime}\left(-\underline{i k}_{\phi}\right), \\
& x^{\prime \prime}\left(\underline{k}_{\phi}\right)=-a^{\prime \prime}\left(-\underline{k}_{\phi}\right) .
\end{aligned}
$$

Equations (D5) and (D6) were ariginally included in the Four-D code for simulating turbulence. The phase-screen model of nondiffraction-1imited beams utilizes the same subroutines.

The following parameters were the basis of an example for comparing the difference between the wavelength-scaling and the phase-screen models of nondiffraction-1imited behavior:

Beam shape

Aperture size, $2 a$

Range

Absorption coeffictent, $\alpha$

Transverse wind speed, $v$

Focal distance, $f$

Beam is $2 x$ diffraction liraited

Wavelength, $\lambda$

Scaled wavelength, $\lambda$

Phase correlation length, $\tau_{0}$

Phase standard deviation, $\sigma_{\phi}$

Number of phase-acreen calculations

for ensamble average
Gaussian

BO cD

$2.5 \mathrm{~km}$

$0.07 \mathrm{~km}^{-1}$

$10 \mathrm{~m} / \mathrm{s}$

$2.5 \mathrm{~km}$

$5.7 \mu \mathrm{m}$

$11.4 \mu \mathrm{m}$

$5.0 \mathrm{~cm}$

$1.177(\mathrm{rad})$

10 
Table Dl gives a comparison of peak intenstties in the focal plane for propagation In vacuum and air. Also included are zesults for a uniformly illuminated aperture of radius $a_{0}=2 a$, which can 1 ikewise be used as a model of a $2 \times$ diffraction-1imited bean.

Table Dl. Comparison of peak intensities in the focal plane. Beam propagates In vacuum (1inear) and air (nonilnear). Case: Gaussian diffractionIimited beaw, a uniformly illuminated aperture (top hat), Gaussian wavelength scaled ( $2 x$ diffraction 1Imited), and a Gaussian beam with a phase screen adjusted to $2 \times$ diffraction 11 mited.

Mode1

Peak Intensity in

focal plane $\left(\mathrm{kW} / \mathrm{cm}^{2}\right)$

Linear

Gaussian, $\lambda$

63.8

Gausslan, $2 \lambda$

15.9

Top hat

17.4

Phase screen

17.7

Nonlinear

Gaussian, $2 \lambda$

Top hat

Phase screen

3.03

The nondiffraction-1imited beams all give roughly one quarter of the focal-plane Intensity of the diffraction-1imited beam when propagated in vacuum. For propagation in a real absorbing atmosphere both the scaled wavelength and the top-hat beam calculations result In twice the peak intensity of the phase-screen model calculation, which is based on an ensemble average taken over 10 independent phise screens. Figures D2 and D3 show respectively the beam intensity as a function of position along the $x$-axis and along a 1lne parallel to the $y$-axis passing through the point of maximum Intensity along the $x$-axis for the wavelength-scaled beam. Figures D4 and DS show the same plots 


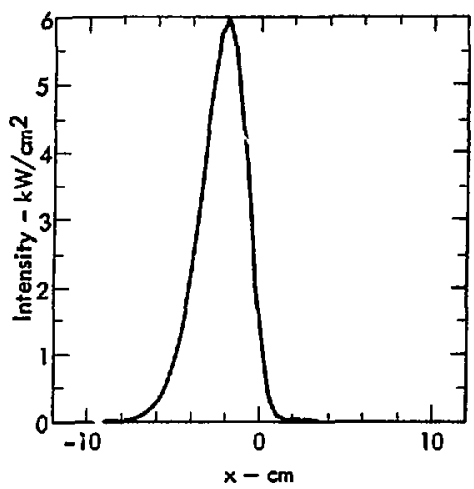

F1g. Dź. Wavelength scaline for $2 \times$ diffraction-limited heam. Intensity on target as a function of $x$ along $x$-axis.

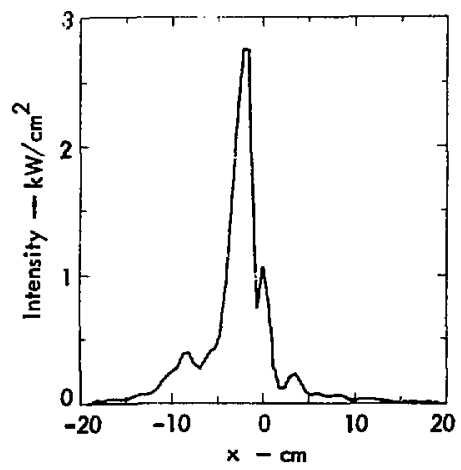

Fig. D4. Phase-screen model of $2 x$ diffraction-1 imited beam averaged over 10 Independent realizations. Intensity on target as a function of $x$ along $x$-axis.

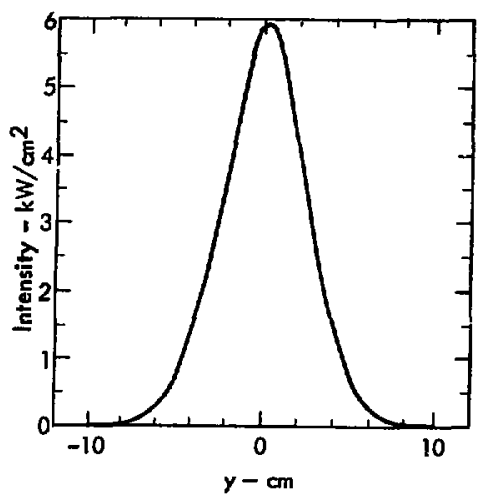

F1g, D3. Wavelength scaling for $2 \times$ diffraction-1imited beam. Intensity on target as a function of $y$ along a line parallel to $y$-axis and passing through point of maximum intensity along the $x$-axis.

for the ensemble averaged phasescreen calculation. In the case of the wavelength-scaled calculation, thermal blooming leads primarily to a broadening of the beam. In the phase-screen calculation, thermal blooming is accompanted by considerable scattering of energy to the far reaches of the mesh.

From these calculations it can be concluded that different models of nondiffraction-1imited beam behavior can lead to qualitatively as well as quantitatively different thermal-blooming behavior. Which 


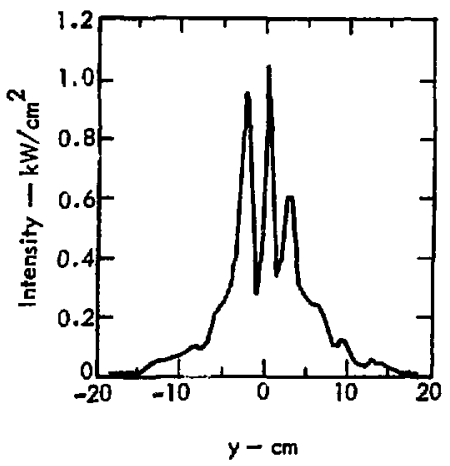

model is best must be determined for each specific altuation. In the last analysis there is no substitute for an accurate experimental characterization of the beam for each specific laser.

F1g. D5. Phase-screen mode1 of $2 \times$ diffraction-1imited beam averaged over 10 ifdependent realizations. Intensity on target as a function of $y$ along a IIne parallel to $y$-axis and passing through point of maximum Intensity along the $x$-axis. 